\title{
THREE LECTURES ON FLAVOUR MIXING
}

Guido Altare11i

CERN - Geneva

\section{CONTENTS}

1. Introduction

2. Basic Formalism

3. General properties of $M_{12}$ and $\Gamma_{12}$

4. The Cabibbo-Kobayashi-Maskawa Matrix

5. Calculation of the Box Diagrams

6. OCD Corrections to Box Diagrams

7. $\overline{\mathrm{B}}^{0}-\overline{\mathrm{B}}^{0}$ Mixing in the Standard Mode 1

8. $\mathrm{B}^{0}-\overline{\mathrm{B}}^{0}$ Mixing beyond the Standard Mode 1

9. Conclusion

Lectures given at the

Cargèse 1987 School on Particle Physics 


\section{INTRODUCTION}

The observation by ARGUS ${ }^{1}$ at DESY of a relatively large amount of $\mathrm{B}^{0}-\overline{\mathrm{B}}^{0}$ mixing, following a previous positive signal of mixing by UAl ${ }^{2}$, was the most important experimental result of the year in particle physics (together with the very recent result on $\varepsilon / \varepsilon$ by the $N A 31^{3}$ collaboration at CERN). The UAl result was already known last year. The ARGUS result refers to the $B_{d}^{0}$ meson $\left(B_{d}^{0} \equiv b \bar{d}, B_{s}^{0} \equiv b \bar{s}\right)$. In terms of $r \equiv P\left(B^{0} \rightarrow B^{0}\right) /$ $P\left(B^{0} \rightarrow B^{0}\right)$, i.e., the ratio of the probability for mixing and for no mixing, ARGUS finds:

$$
r_{d}=0.21 \pm 0.08
$$

The experimental method was described in the lectures by S.L. Wu ${ }^{4}$. on the theoretical side a large number of papers have been devoted on $B^{0}-\bar{B}^{0}$ mixing in the past 5 and then recently $6-10$ after the UAl and ARGUS results. These lectures are intended to an elementary introduction to flavour mixing in general and to $B^{0}-\bar{B}^{0}$ mixing in particular. Their purpose is to provide the reader with the essential background necessary to follow the current specialized literature.

\section{BASIC FORMALISM}

For a stable free particle at rest the quantum mechanics time evolution is given by $\psi \sim e^{-i M t}$ For an unstable particle at rest, this is modified into $\psi \sim \mathrm{e}^{-i(M-i \Gamma / 2)}$ t (in fact, $\left.|\psi|^{2} \sim \mathrm{e}^{-\Gamma t}=\mathrm{e}^{-\mathrm{t}}\right)_{\tau}$ ), with $M$ and $\Gamma$ real, positive numbers. For several coupled states $M$ and $\Gamma$ became Hermitian matrices with positive eigenvalues (i.e., the analogue of real, positive numbers). In particular for $B^{0}-\bar{B}^{0}$ (or any other similar system), we have:

$$
\text { "H"( } \left.\frac{B^{0}}{B^{0}}\right)=\left(\begin{array}{ll}
M-i \Gamma / 2 & M_{12}-i \Gamma_{12 / 2} \\
M_{12}^{*}-i \Gamma_{12 / 2}^{*} & M-i \Gamma / 2
\end{array}\right)\left(\begin{array}{l}
B^{0} \\
B^{0}
\end{array}\right)
$$

Note that a) " $\mathrm{H}$ " is not Hermitian (since probability is not conserved within the $\mathrm{B}^{0}-\overrightarrow{\mathrm{B}}^{0}$ system, because of the decays); $b$ ) $\mathrm{H}_{1} \downarrow=\mathrm{H}_{22}$ by CPT; 
c) $\mathrm{H}_{12} \neq 0, \mathrm{H}_{21} \neq 0$ because of the weak interactions which violate the conservation of quark flavours. d) Im $M_{12} \neq 0$, Im $\Gamma_{12} \neq 0$ because of CP violation.

The eigenvalues of " $H$ " can be written down in the form:

$$
B_{1,2}=\frac{(1+\epsilon) B^{0} \pm(1-\epsilon) \bar{B}^{0}}{\sqrt{2\left(1+|\epsilon|^{2}\right)}}
$$

Note that $B_{1}$ and $B_{2}$ are not orthogonal because " $H$ " is not Hermitian. If $\varepsilon=0, \mathrm{CP}$ is conserved in the wave functions. In general, $\varepsilon$ depends on the phase convention chosen. Thus, for example, $\varepsilon$ pure imaginary does not lead to any $C P$ violation because it $c$ an be removed by a redefinition of the relative $\mathrm{B}^{0}-\bar{B}^{0}$ phase. A simple calculation immediately leads to the following results for $\varepsilon$ and the eigenvalues of $M$ and $\Gamma$ :

$$
\begin{aligned}
\eta \equiv \frac{1-\epsilon}{1+\epsilon} & =\sqrt{\frac{M_{12}^{*}-i \Gamma_{12 / 2}^{*}}{M_{12}-i \Gamma_{12} / 2}} \\
M_{1,2} & =M \pm \operatorname{Re} Q \\
\Gamma_{1,2} & =\Gamma \mp 2 \operatorname{Im} Q
\end{aligned}
$$

where $M, \Gamma, M_{12}$ and $\Gamma_{12}$ are defined in Eq. (2.1) and

$$
Q=\sqrt{\left(M_{12}-i \Gamma_{12 / 2}\right)\left(M_{12}^{*}-i \Gamma_{12 / 2}^{*}\right)}
$$

$B^{0}-\bar{B}^{0}$ oscillations are caused by the different evolution in time of the eigenvectors $B_{1}$ and $B_{2}$. Starting at $t=0$ from a pure $B^{0}$ state:

$$
|\psi| t=0|\rangle=\left|B^{0}\right\rangle=\left(\left|B_{1}\right\rangle+\left|B_{2}\right\rangle\right) \frac{\sqrt{1+|\epsilon|^{2}}}{\sqrt{2}(1+\epsilon)}
$$

one obtains at time $t$ :

$$
|\psi(t)\rangle=\frac{\sqrt{1+|\epsilon|^{2}}}{\sqrt{2}(1+\epsilon)}\left[\left|B_{1}\right\rangle e^{-i\left(M_{1}-\frac{i}{2} \Gamma_{1}\right) t}+\left|B_{2}\right\rangle e^{-i\left(M_{2}-\frac{i}{2} \Gamma_{2}\right) t}\right]
$$

By using Eq. (2.2) we can eliminate $\left|B_{1}\right\rangle$ and $\left|B_{2}\right\rangle$ and write $|\psi(\varepsilon)\rangle$ as a superposition of $B^{0}$ and $\bar{B}^{0}$. The coefficients are the transition amplitudes: $A(B \rightarrow B)$ and $A(B \rightarrow B)$. One immediately obtains:

$$
\begin{aligned}
& A(B \rightarrow B)=\frac{1}{2}\left[e^{-i M_{1} t} e^{-\frac{1}{2} \Gamma_{1} t}+e^{-i M_{2} t} e^{-\frac{1}{2} \Gamma_{2} t}\right] \\
& A(B \rightarrow \bar{B})=\frac{1-\varepsilon}{1+\epsilon} \frac{1}{2}\left[e^{-i M_{1} t} e^{-\frac{1}{2} \Gamma_{1} t}-e^{-i M_{2} t} e^{-\frac{1}{2} \Gamma_{2} t}\right]
\end{aligned}
$$
lities:

We define the ratio $r$ of total (i.e., integrated over time) probabi-

$$
r=\frac{P(B \rightarrow \bar{B})}{P(B \rightarrow B)}=\frac{\int_{0}^{T}|A(B \rightarrow \bar{B})|^{2} d t}{\int_{0}^{T}|A(B \rightarrow B)|^{2} d t}
$$

where $T$ is a conveniently large time. One directly obtains from Eqs. (2.9) and $(2.10)$ : 
$r=\left|\frac{1-\epsilon}{1+\epsilon}\right|^{2} \frac{\int_{0}^{T} d t\left[e^{-\Gamma_{1} t}+e^{-\Gamma_{2} t}-2 e^{-\Gamma t} \cos (\Delta m t)\right]}{\int_{0}^{T} d t\left[e^{-\Gamma_{1} t}+e^{-\Gamma_{2} t}+2 e^{-\Gamma t} \cos (\Delta m t)\right]}$

where $\Gamma=\frac{1}{2}\left(\Gamma_{1}+\Gamma_{2}\right)$ is the average width of $B_{1}$ and $B_{2}$ and $\Delta m=M_{1}-M_{2}$ is their mass difference (we define 1 and 2 such that $\Delta m>0$ ). By performing the integrals for $T \rightarrow \infty$, we finally obtain:

$$
r=\left|\frac{1-\epsilon}{1+\epsilon}\right|^{2} \frac{x^{2}+y^{2}}{2+x^{2}-y^{2}}
$$

where $\mathrm{x}=\Delta \mathrm{m} / \Gamma$ and $\mathrm{y}=\Delta \Gamma / 2 \Gamma$ with $\Delta \Gamma=\Gamma_{1}-\Gamma_{2}$. Similarly, we could have obtained

where

$$
\bar{r}=\left|\frac{1+\epsilon}{1-\epsilon}\right|^{2} \frac{x^{2}+y^{2}}{2+x^{2}-y^{2}}
$$

$$
\bar{r} \equiv \frac{P(\bar{B} \rightarrow B)}{P(\bar{B} \rightarrow \bar{B})}
$$

Clearly, when CP violation effects are neglected

so that the as ymmetry

$$
r=\bar{r}=\frac{x^{2}+y^{2}}{2+x^{2}-y^{2}}
$$

$$
a=\frac{r-\bar{r}}{r+\bar{r}}
$$

is a we11-known measure of $\mathrm{CP}$ violation. Note that

$$
\begin{aligned}
& 0 \leqslant x^{2} \leqslant+\infty \\
& 0 \leqslant y^{2}=\left(\frac{\Gamma_{1}-\Gamma_{2}}{\Gamma_{1}+\Gamma_{2}}\right)^{2} \leqslant 1
\end{aligned}
$$

Then, neglecting $C P$ violation, we have

$$
0 \leqslant r \leqslant 1
$$

An alternative parameter for $B-\bar{B}$ mixing, often used, is $\chi$ :

$$
r=\frac{x}{1-\chi} \text { or } \chi=\frac{r}{1+r}
$$

From Eq. (2.18), it follows that $0<\chi<\frac{1}{2}$.

Typically $\mathrm{r}$ is measured through the ratio:

$$
\rho=\frac{N(B B)+N(\bar{B} \bar{B})}{N(B \bar{B})+N(\bar{B} B)}
$$

where $N(B B)$ is the number of $B B$ final states observed in a sample of events from a process where a $B \bar{B}$ pair is produced. $N(B B)$ is identified by some convenient final state (e.g., two negatively charged leptons: $\left.\ell^{-} \ell^{-}\right) . \quad N(B \bar{B})$ and $N(\vec{B} B)$ are experimentally the same but are kept separate to remind us of the possibility of a double flip.

If the $B \bar{B}$ pair is produced incoherently (i.e., in each event the angular momentum, parity, etc., of the BB system are different) then, neglecting $C P$ violation: 


$$
\rho=\frac{2 r}{1+r^{2}}
$$

because $N(B B)=N(\bar{B} \bar{B}) \sim \chi(1-\chi), N(B \bar{B}) \sim(1-\chi)^{2}, N(\bar{B} B) \sim \chi^{2}$ where $\chi$ is the flip probability given in Eq. (2.19).

But if the $B \bar{B}$ pair is produced coherently (for example, at the $T(4 S)$ $B \bar{B}$ has $J \equiv 1, C=-1$, etc.) then $\rho$ is different. For $J$ odd, one obtains

$$
\rho=r \quad \text { (J odd) }
$$

This is because quantum mechanics is at work. Let us see how ${ }^{l l}$.

Coherence with $\mathrm{J}=1, \mathrm{C}=-1$ means that the produced state is:

$$
\left|B\left(k_{1}, t\right)\right\rangle\left|\bar{B}\left(k_{2}, t\right)\right\rangle-\left|\bar{B}\left(k_{1}, t\right)\right\rangle\left|B\left(k_{2}, t\right)\right\rangle
$$

with (we always neglect $C P$ violation):

$$
\begin{aligned}
& |B(t)\rangle=R(t)|B\rangle+C(t)|\bar{B}\rangle \\
& |\bar{B}(t)\rangle=C(t)|B\rangle+R(t)|\bar{B}\rangle
\end{aligned}
$$

$R(t), C(t)$ are the amplitudes to "remain" or to "change", i.e., $R=A(B \rightarrow B)=A(\bar{B}>\bar{B})$ and $C=A(B \rightarrow \bar{B})=A(\bar{B}>B)$. Here $|B(t)\rangle$ means the state that evolves with time starting from $|B\rangle$ at $t$ ime $t=0$. If the two decays take place at the times $t_{1}$ and $t_{2}$, the corresponding superpositions of states are given by:

$$
\begin{aligned}
& \left(R_{1} C_{2}-C_{1} R_{2}\right)|B B\rangle+\left(R_{1} R_{2}-C_{1} C_{2}\right)|B \bar{B}\rangle+ \\
& +\left(C_{1} C_{2}-R_{1} R_{2}\right)|\bar{B} B\rangle+\left(C_{1} R_{2}-C_{2} R_{1}\right)|\bar{B} \bar{B}\rangle
\end{aligned}
$$

where $R_{1} C_{2}$ mean $R\left(t_{1}\right) C\left(t_{2}\right)$, etc. Now:

$$
\rho=\frac{\int\left|R_{1} c_{2}-c_{1} R_{2}\right|^{2} d t_{1} d t_{2}}{\int\left|R_{1} R_{2}-c_{1} c_{2}\right|^{2} d t_{1} d t_{2}}=\frac{\operatorname{NUM}(\rho)}{\operatorname{DEN}(\rho)}
$$

where $R$ and $C$ are directly obtained from Eqs. (2.9). With few steps one obtains:

$$
\begin{aligned}
& \operatorname{DEN}(\rho)=\int\left[e^{-\Gamma_{1} t_{1}} e^{-\Gamma_{2} t_{2}}+e^{-\Gamma_{2} t_{1}} e^{-\Gamma_{1} t_{2}} \pm\right. \\
& \left.\quad \pm 2 \operatorname{RUM}(\rho) e^{-\Gamma t_{1}} e^{i \Delta m t_{1}} e^{-\Gamma t_{2}} e^{-i \Delta m t_{2}}\right] d t_{1} d t_{2}(2.27) \\
& =\frac{2}{\Gamma_{1} \Gamma_{2}} \pm 2 \operatorname{Re} \frac{1}{\Gamma-i \Delta m} \frac{1}{\Gamma+i \Delta m}=\frac{2}{\Gamma^{2}-\frac{\Delta \Gamma^{2}}{4}} \pm \frac{2}{\Gamma^{2}+\Delta m^{2}} \\
& \text { Thus, one finally gets: }
\end{aligned}
$$




$$
\rho=\frac{\Delta m^{2}+\Delta n^{2} / 4}{2 \Gamma^{2}+\Delta m^{2}-\Delta \Gamma^{2} / 4}=r
$$

\section{GENERAL PROPERTIES OF $M_{12}$ and $\Gamma_{12}$}

A double change of flavour, $\Delta b=2$, as in $B^{0}-\bar{B}^{0}$ mixing, clearly requires the action of two charged currents. The two emitted $W^{\prime} s$ must be reabsorbed, so that a total of four weak vertices are involved. Quite in general, including all effects of strong interactions, one can write:

$$
M_{12}=\int d x d y d z D_{\mu \nu}(x-y) D_{\rho}(z)\left\langle B^{\circ}\left|J^{\mu}(x) J^{\nu}(y) J^{\rho}(z) J^{\sigma}(0)\right| \bar{B}^{0}\right\rangle(3.1)
$$

where $D_{\mu y}$ is the $W$ propagator. In terms of Feynman diagrams, the above formula includes box diagrams with all possible gluon exchanges among quark legs. This is clearly a very complicated object. The situation would become simpler if one could exploit the fact that QCD is asymtotically free at short distances. The effective interaction induced by $E q$. (3.1) involves four space-time points where the $W^{\prime} s$ are emitted or reabsorbed. If one can prove that the four points at some level of approximation can be confused with a single point, then the effective interaction becomes local. The QCD effects can then, at least in principle, be taken into account perturbatively. The ideal situation is for mext $<$ $m_{Q} \ll M_{W}$, where $m_{e x t}$ is the external meson mass and $m_{Q}$ is the exchanged virtual quark mass. The $W$ propagator $D_{\mu \nu}(u)$ is significantly different from zero only at distances $u \lesssim 1 / M_{W}$.

On the other hand, at short distances $x-y \leqslant 1 / M_{W}$ an operator expansion is valid, and $J_{\mu}(x) J^{\mu}(y)$ approaches a local form fermion operator. Thus the four-point function in Eq. (3.1) can be reduced to a two-point function when terms of order $\mathrm{m}_{\text {ext }}^{2} / \mathrm{M}_{\mathrm{W}}^{2}, \mathrm{~m}_{\mathrm{Q}}^{2} / \mathrm{M}_{\mathrm{W}}^{2}$ are neglected. For heavy enough virtual quarks the operator expansion technique $c$ an in an analogous way be applied to the resulting product of two local four fermion operators. The heavy virtual quark lines can also be shrunk down to a point. The effective Hamiltonian is then finally given in terms of a single local four fermion operator:

$$
H_{e f f} \approx G_{F}^{2} \bar{d}_{L} \gamma_{\mu} b_{L} \bar{d}_{L} \gamma^{\mu} b_{L} c\left(m_{b}, m_{Q}, M_{W}, \ldots\right)
$$

where

$$
q_{L}=\frac{1-\gamma_{5}}{2} q
$$

and $c$ is a coefficient function. Since at short distance the QCD coupling $\alpha_{s}$ is small, one can compute the coefficient function $c$ in the approximation of neglecting the strong interactions. This corresponds to the evaluation of the box diagrams (Fig. 1). The QCD corrections are then, in principle, computable and are determined by the anomalous dimensions of the different operators which enter in the short distance expansion (see Section 6). When $\mathrm{m}_{\mathrm{Q}} 2 \mathrm{M}_{\mathrm{W}}$ the dominance of short distances remains true. What is to be rediscussed is the appropriate 1 imiting procedure (e.g., $\mathrm{M}_{\mathrm{W}}, \mathrm{m}_{\mathrm{Q}} \rightarrow \infty$ with $\mathrm{m}_{\mathrm{Q}} / \mathrm{M}_{\mathrm{W}}$ fixed).

In order for the above strategy to apply, it is necessary to have an argument for the dominance of heavy virtual quarks in the case of interest. As we shall see, it is the structure of the quark mixing matrix that fixes the relative importance of the different virtual quark 
exchanges. For example, for $B_{d}^{0}-B_{d}^{0}$ mixing, $M_{12}$ is dominated by the top quark exchange, because $\left|v_{t b} v_{t d}^{*}\right|$ is of the same order of $\left|v_{c b} v_{c d}^{*}\right|$. The same is also true for $B_{S}^{0}-\bar{B}_{s}^{0}$ mixing. On the other hand, $M_{1} 2$ would vanish if all exchanged quarks had the same mass, due to the GIM mechanism 12 ( $b$ and $d$ quarks are coupled to orthogonal combinations of $u, c$ and $t$ quarks). Thus for $B^{0}-\bar{B}^{0}$ mixing the ratio of $t$ to $c$ contributions is of order $\mathrm{m}_{\mathrm{t}}^{2} / \mathrm{m}_{\mathrm{c}}^{2}$.

Even if, in a given case, the short distance expansion can be applied, one is still confronted with the problem of computing the matrix element of $\mathrm{H}_{\text {eff }}$ in Eq. (3.2) between the external meson-antimeson states. This is a different problem. It is precisely at this stage that we cannot avoid to cope with QCD at scales of order mext. Thus, if the external meson is light (e.g., the kaon) the problem is not easy. One tentative solution is to evaluate the matrix element by vacuum insertion (or saturation) 13. This is a kind of valence approximation which will be discussed in section 5. The vacuum saturation approximation is expected to work better and better as the mass of the external meson increases. This is because of the Zweig rule: gluon radiation from the quark legs is suppressed when the relevant transverse momentum of order of the meson mass is large.

In the standard model all complex phases enter through the CabibboKobayashi-Maskawa (CKM) matrix (see Fig. l). The phases determine Im $M_{12}$ vs. $\operatorname{Re} M_{12}$ and $I n \Gamma_{12}$ vs. Re $\Gamma_{12}$.

$\Gamma_{12}$ is given by the absorptive part of the diagrams of Fig. 2. A cut indicates the on-shell particles of the final state after decay (with the integrations over the available phase space). The cut in Fig. 2a corresponds to spectator decays, while the cut in Fig. $2 b$ has to do with $W$-exchange decay modes. Clearly only common final states for $B$ and $\bar{B}$ decay contribute to $\Gamma_{12}$. Examples of common final states are given in Fig. 3. Obviously, only light quarks can appear in the final state. In the 1 imit when all quarks are massless except for $b$ and $t$ quarks, $\Gamma_{12}$ for $B$ mesons turns out to be proportional to $\mathrm{m}_{b}^{2}$. Actually, in this limit

$$
\Gamma_{12} \propto\left[V_{u b} V_{u d}^{*}+V_{c b} V_{c d}^{*}\right]^{2} m_{b}^{2}=\left(V_{t b} V_{t d}^{*}\right)^{2} m_{b}^{2}
$$

where the last equality is due to the unitarity of the $3 \times 3$ CKM matrix.

Two important consequences follow for $B$ mesons: a) $\Gamma_{12}$ has almost the same phase of $M_{12}$ (both being determined by $v_{t b} v_{t d}^{*}$ ); b) $\left|\Gamma_{12}\right|<<$ $\left|\mathrm{M}_{12}\right|$ or $\Delta \Gamma \ll \Delta \mathrm{m}$ [see Eq. (2.6)] in the same proportion as $\mathrm{m}_{\mathrm{b}}^{2} \ll \mathrm{m}_{\mathrm{t}}^{2}$. Thus for $B$ mesons a quite good approximation is

$$
r=\frac{x^{2}}{2+x^{2}}
$$

Also in the limit that $M_{12}$ and $\Gamma_{12}$ have the same phase, $\varepsilon$ is purely imaginary as seen from Eq. (2.3). If $M_{12}=\left|M_{12}\right| e^{i \phi}, \Gamma_{12}=\left|\Gamma_{12}\right| e^{i \phi}$, then

$$
\frac{1-\epsilon}{1+\epsilon}=e^{-i \varphi}
$$

In this case Re $\varepsilon=0$ and the effect of $\varepsilon$ can be rotated away by a phase redefinition. Thus, there are two ways of having small effects of CP violation: either $\operatorname{Im} M_{12}, \operatorname{Im} \Gamma_{12}$ are small, then $|\varepsilon|$ is sma11; or $M_{12}$ 


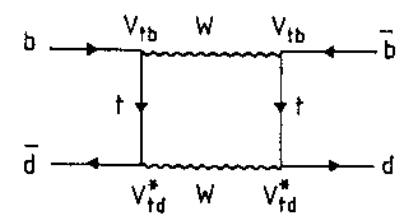

a)

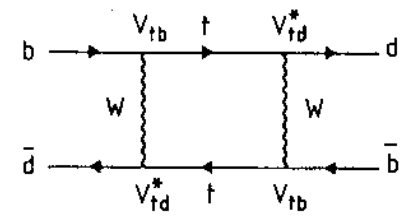

b)

Fig. 1 Box diagrams for $\mathrm{B}_{\mathrm{d}}^{0}-\overline{\mathrm{B}}_{\mathrm{d}}^{0}$ mixing with top quark exchange.
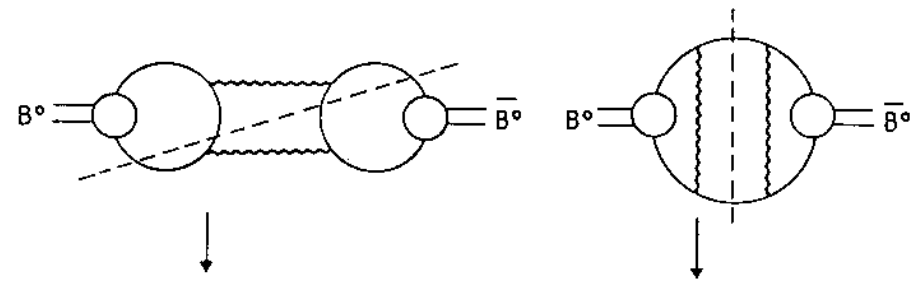

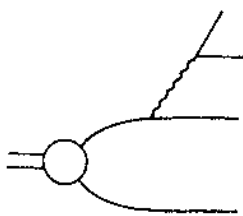

Spectator

a)

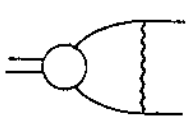

Annihilation

b)

Fig. 2 Contribution to $\Gamma_{12}$ for the $B^{0}-\bar{B}^{0}$ system.
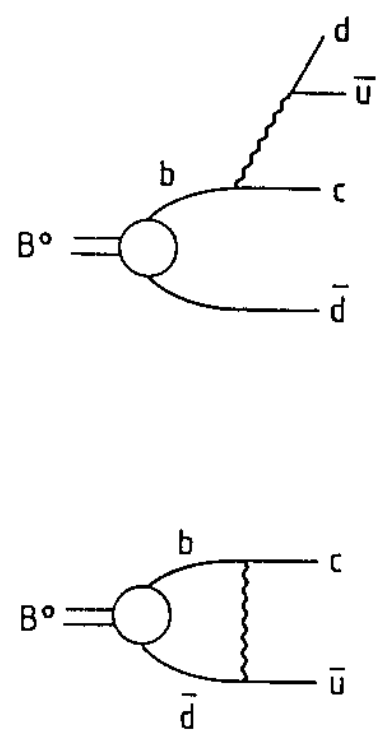
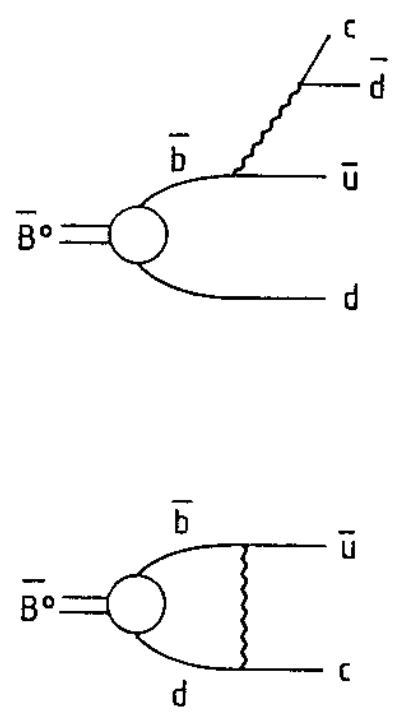

Fig. 3 Examples of common final states in $\mathrm{B}^{0}$ and $\overline{\mathrm{B}}^{0}$ decays. 
and $\Gamma_{12}$ can have nearly the same phase. In the CKM phase convention the first option is realized for the kaon system, the second for the $B$ meson system.

Going back to Eqs. $(2.3)-(2.6)$, we see that in general

$$
\begin{aligned}
& \Delta m=2 \operatorname{Re} Q \\
& \Delta \Gamma=-4 \operatorname{Im} Q
\end{aligned}
$$

For $B$ mesons $M_{12}$ and $\Gamma_{12}$ have nearly the same phase. Then

$$
Q \simeq\left|M_{12}\right|-\frac{i}{2}\left|\Gamma_{12}\right|
$$

so that

$$
\begin{aligned}
& \Delta m_{B} \simeq 2\left|M_{12}\right| \\
& \Delta \Gamma_{B} \simeq 2\left|\Gamma_{12}\right|
\end{aligned}
$$

For kaons the situation is different as we shall now summarize.

For kaons ${ }^{18}$ :

$$
\begin{aligned}
& \Delta m_{K}=m_{K_{L}}-m_{K_{s}}=(3.521 \pm 0.014) 10^{-12} \mathrm{MeV} \\
& \tau_{s}=(0.8923 \pm 0.0022) 10^{-10} \mathrm{~s} \\
& \tau_{L}=(0.5183 \pm 0.0040) 10^{-7} \mathrm{~s}
\end{aligned}
$$

As a consequence of $\Gamma_{S} \gg \Gamma_{L}$ one has

$$
\begin{aligned}
& \Gamma_{K}=\frac{1}{2}\left(\Gamma_{s}+\Gamma_{L}\right) \simeq \frac{1}{2} \Gamma_{S} \\
& \Delta \Gamma_{K}=\Gamma_{L}-\Gamma_{S} \simeq-\Gamma_{S}
\end{aligned}
$$

and $y_{\mathrm{K}}=\Delta \Gamma / 2 \Gamma \simeq-1$ (actually $\left.\mathrm{y}_{\mathrm{K}}^{2} \simeq 1-4 \Gamma_{\mathrm{L}} / \Gamma_{\mathrm{S}} \sim 1-7 \cdot 10^{-3}\right)$. Also

Final1y

$$
x_{k} \simeq \frac{\Delta m_{k}}{\Gamma_{k}} \sim 0.954
$$

$$
r_{k} \simeq \frac{x_{k}^{2}+y_{k}^{2}}{2+x_{k}^{2}-y_{k}^{2}} \simeq 1-7 \cdot 10^{-3}
$$

Moreover, for the kaon system the CP violation parameter $|E|$ is given by :

$$
|E| \simeq(2.28 \pm 0.02) \cdot 10^{-3}
$$

The small absolute value of $\varepsilon$ implies that for kaons

$$
\begin{aligned}
& \operatorname{Im} M_{12} \ll \operatorname{Re} M_{12} \\
& \operatorname{Im} \Gamma_{12} \ll \operatorname{Re} \Gamma_{12}
\end{aligned}
$$

because $\varepsilon=0$ if $M_{12}$ and $\Gamma_{12}$ are both real [Eq. (2.3)]. From Eqs. $(2.4)-(2.5)$ we see that for kaons

$$
\begin{aligned}
& \Delta m_{k}=2 \operatorname{Re} M_{12} \\
& \Delta \Gamma_{k} \simeq 2 \operatorname{Re} \Gamma_{12}
\end{aligned}
$$

Note the difference with Eq. (3.9) valid for the B system.

Starting from the general expression for $\varepsilon$ given by Eq. (2.3) we can expand in the small imaginary parts $\operatorname{Im} M_{12}, \operatorname{Im} \Gamma_{12}$ : 


$$
\begin{aligned}
\frac{1-\epsilon}{1+\epsilon} & \simeq 1-2 \epsilon=\sqrt{\frac{\operatorname{Re} M_{12}-i \operatorname{Im} M_{12}-\frac{i}{2} \operatorname{Re} \Gamma_{12}-\frac{1}{2} \operatorname{Im} \Gamma_{12}}{\operatorname{Re} M_{12}+i \operatorname{Im} M_{12}-\frac{i}{2} \operatorname{Re} \Gamma_{12}+\frac{1}{2} \operatorname{Im} \Gamma_{12}}} \\
& \simeq 1-\frac{i \operatorname{Im} M_{12}+\frac{1}{2} \operatorname{Im} \Gamma_{12}}{\operatorname{Re} M_{12}-\frac{i}{2} \operatorname{Re} \Gamma_{12}}
\end{aligned}
$$

Thus, for kaons:

$$
\epsilon \simeq \frac{i \operatorname{Im} M_{12}+\frac{1}{2} \Gamma_{12}}{\Delta m-i \Delta \Gamma / 2}
$$

We have al ready seen that $\Delta \mathrm{m}_{\mathrm{K}} \simeq-\Delta \Gamma_{\mathrm{K}} / 2$. Also for kaons $\mathrm{Im} \Gamma_{\mathrm{K}} \simeq 0$. This follows because only u quark can contribute to kaon decay (as opposed to $c$ and $t$ quarks). But $V_{u d}$ and $V_{u s} c a n$ be chosen to be real, so that Im $\Gamma_{\mathrm{K}} \simeq 0$ for kaons. Hence, one obtains

$$
\epsilon \simeq e^{i \pi / 4} \frac{\operatorname{Im} M_{12}}{\sqrt{2} \Delta m}
$$

Actually the experimental definition of $\varepsilon$ is different than that given by Eq. (2.2) because of a different phase convention. The measured value of $\varepsilon[\mathrm{Eq} .(3.14)]$ corresponds to the definition:

$$
\begin{aligned}
& \eta_{+-}=\frac{A\left(k_{L} \rightarrow \pi^{+} \pi^{-}\right)}{A\left(k_{S} \rightarrow \pi^{+} \pi^{-}\right)}=\epsilon+\epsilon^{\prime} \\
& \eta_{00}=\frac{A\left(k_{L} \rightarrow \pi^{\circ} \pi^{\circ}\right)}{A\left(k_{S} \rightarrow \pi^{\circ} \pi^{\circ}\right)}=\epsilon-2 \epsilon^{\prime}
\end{aligned}
$$

The two definitions of $\varepsilon$ coincide for the phase choice $\operatorname{Im} A_{0}=0$, where $A_{I}=\left|A_{I}\right| e^{1} \delta_{I}=A\left(K^{0} \rightarrow(\pi \pi)_{I}\right), I=0,2$ being the isospin. Instead, the phase definition chosen here was specified by the requirement that $\left|K^{0}\right\rangle=$ $\mathrm{CP}\left|\overline{\mathrm{K}}^{0}\right\rangle$. By defining:

$$
\xi=\frac{\operatorname{Im} A_{0}}{\operatorname{Re} A_{0}}
$$

the correction to Eq. (3.17) is given by ${ }^{14}$ :

Similarly

$$
E=\frac{e^{i \pi / 4}}{\sqrt{2}}\left[\frac{I m M_{12}}{\Delta m}+\xi\right]
$$

$$
\frac{\epsilon^{\prime}}{\epsilon}=\frac{1}{\sqrt{2}} \frac{1}{\epsilon} e^{i\left(\delta_{2}-\delta_{0}+\frac{\pi}{2}\right)}\left[\frac{\operatorname{Im} A_{2}}{\operatorname{Re} A_{0}}-\frac{\operatorname{Re} A_{2}}{\operatorname{Re} A_{0}} \xi\right]
$$

Note that experimentally $\delta_{2}-\delta_{0}+\pi / 2 \approx(48 \pm 8)^{\circ}$ so that $e^{i\left(\delta_{2}-\delta_{0}+\pi / 2\right)} \approx$ $\mathrm{e}^{1 \pi / 4}$. The experimental validity of the $\Delta \mathrm{I}=\frac{2}{2}$ rule implies:

$$
\omega=\frac{\operatorname{Re} A_{2}}{\operatorname{Re} A_{0}} \simeq 0.045
$$


According to Eq. (3.23), $\varepsilon^{\prime}$ is determined by the imaginary parts of the $\Delta S=1 \mathrm{~K} \rightarrow \pi \pi$ amplitudes. These are zero in the standard model at tree level. At one loop, imaginary parts are generated by the famous penguin diagrams, Fig. 4. These diagrams only contribute to the $\Delta I=\frac{1}{2}$ amplitudes (hence to $A_{0}$ and not to $A_{2}$ ) so that $\operatorname{Im~} A_{2}=0$. Thus

$$
\left.\left|\frac{E^{\prime}}{\epsilon}\right|=\frac{1}{\sqrt{2} \epsilon} \omega|\xi| \simeq 14 \mid\right\} \mid
$$

( $\varepsilon^{\prime} / \varepsilon$ is nearly real and is positive for $\xi$ negative, as is most probably the case in the standard model).

The very recent experimental result by the NA31 Collaboration ${ }^{3}$ at CERN provides us with the value:

or

$$
\frac{\epsilon^{\prime}}{\epsilon}=(3.5 \pm 0.7 \pm 0.4 \pm 1.2) 10^{-3}
$$

$$
\frac{\epsilon^{\prime}}{\epsilon}=(3.5 \pm 1.5) 10^{-3}
$$

if the errors are added in quadrature.

\section{THE CABIBBO-KOBAYASHI-MASKAWA MATRIX}

Given the experimental values of the quark mixing angles, the most convenient parametrization of the CKM matrix is the one proposed by Maianil5. In comparison, the original proposal by Kobayashi-Maskawal6 leads to cumbersome expressions for the physically relevant transition amplitudes.

We denote by $d^{\prime}, s^{\prime}, b^{\prime}$ the three down partners of $u, c$, $t$ respectively in the weak charged current left doublets. We write, following Maiani,

$$
\left|d^{\prime}\right\rangle=c_{\beta}\left|d_{c}\right\rangle+s_{\beta} e^{i \varphi}|b\rangle
$$

where $c_{\beta} \equiv \cos \beta, s_{\beta} \equiv \sin \beta$ (analogous shorthand notations will be used in the following). $d_{c}^{\beta}$ is the Cabibbo rotated down quark

$$
\left|d_{c}\right\rangle=c_{\theta}|d\rangle+S_{\theta}|s\rangle
$$

Note that in a four quark model the Cabibbo angle fixes both the ratio of the $u$ to $d$ coupling with respect to the $\nu_{\mu}$ to $\mu$ coupling and the $r$ atio of the $u$ to $s$ and $u$ to $d$ couplings. In a six quark model one has to choose whether to keep the first or the second definition. Here the second is taken and in fact the $u$ to $d$ coupling is given by $\cos \beta \cos \theta_{c}$, i.e., it is no longer completely specified by $\theta_{c}$. Al so note that we can certainly fix the phases of $u, d, s$ and $b$ so that a real coefficient appears in front of $\mathrm{d}_{\mathrm{c}}$.

We now construct two orthonormal vectors, both orthogonal to d'. They can be chosen as the Cabibbo rotated strange quark:

$$
\left|s_{c}\right\rangle=-s_{\theta}|d\rangle+c_{\theta}|s\rangle
$$

and as

$$
|v\rangle=-s_{\beta} e^{-i \varphi}\left|d_{c}\right\rangle+c_{\beta}|b\rangle
$$




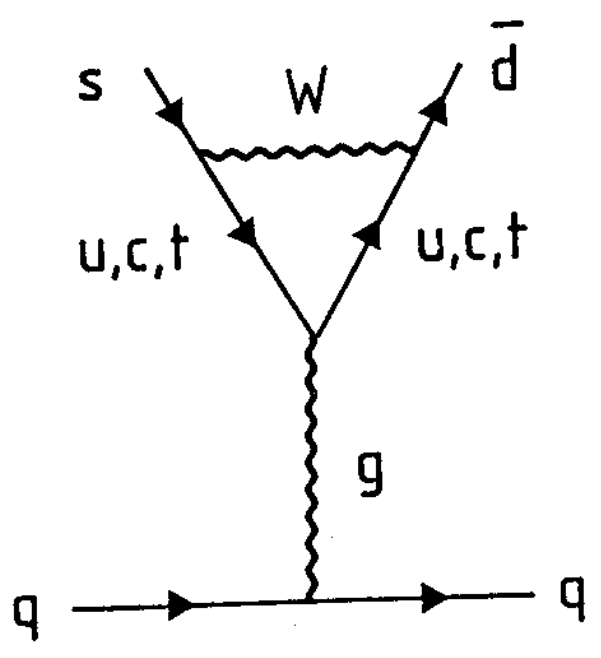

Fig. 4 Penguin diagram with gluon exchange. 
The angle $\gamma$ is now defined by the physical combinations of $s_{c}$ and $v$ coupled to $c$ and $t$ quarks:

$$
\begin{aligned}
& \left|s^{\prime}\right\rangle=c_{\gamma}\left|s_{c}\right\rangle+s_{\gamma}\left(-s_{\beta} e^{-i \varphi_{1}}\left|d_{c}\right\rangle+c_{\beta}|b\rangle\right) \\
& \left|b^{\prime}\right\rangle=-s_{\gamma}\left|s_{c}\right\rangle+c_{\gamma}\left(-s_{\beta} e^{-i \varphi}\left|\partial_{c}\right\rangle+c_{\beta}|b\rangle\right)
\end{aligned}
$$

From experiment

$$
s_{\theta} \equiv \lambda=0.221 \pm 0.002
$$

Also $s_{\gamma} \sim \lambda^{2}$ and $s_{\beta} \sim \lambda^{3}$. Thus, empirically $s_{\gamma}$ and $s_{\beta}$ are very small in the Malani convention. Neglecting terms of order $\lambda^{4}$, one obtains a

$\left(\begin{array}{c}|u\rangle \\ \left|d_{c}\right\rangle+s_{\beta} e^{i \varphi}|b\rangle\end{array}\right)\left(\begin{array}{c}|c\rangle \\ \left|s_{c}\right\rangle+s_{\gamma}|b\rangle\end{array}\right)\left(\begin{array}{c}|t\rangle \\ |b\rangle-s_{\gamma}\left|s_{c}\right\rangle-s_{\beta} e^{-i \varphi}|d\rangle\end{array}\right)(4.7)$

or

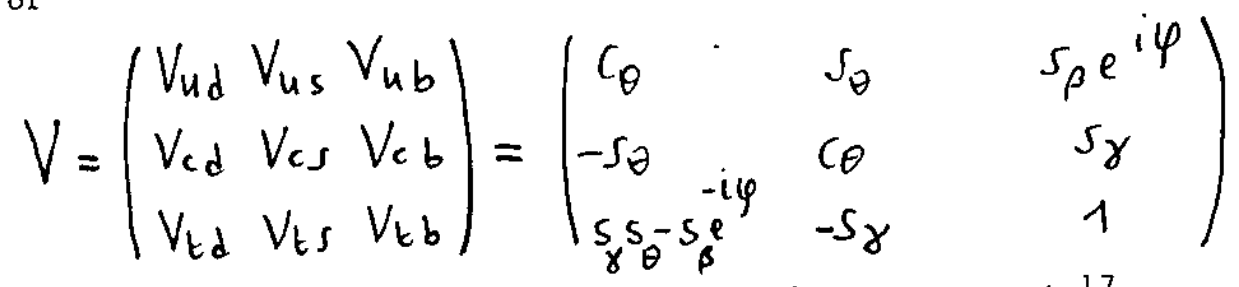

Finally, it is convenient to set, following wolfenstein 17

$$
\begin{aligned}
& s_{\gamma}=A \lambda^{2} \\
& s_{\beta}=A \lambda^{3} \rho
\end{aligned}
$$

Then $V$ takes the simple form

$$
V=\left\{\begin{array}{cc}
1-\frac{\lambda^{2}}{2} & \lambda \\
-\lambda & 1-\frac{\lambda^{2}}{2} \\
A \lambda^{3}\left(1-\rho e^{-i \varphi}\right) & -A \lambda^{2}
\end{array}\right.
$$

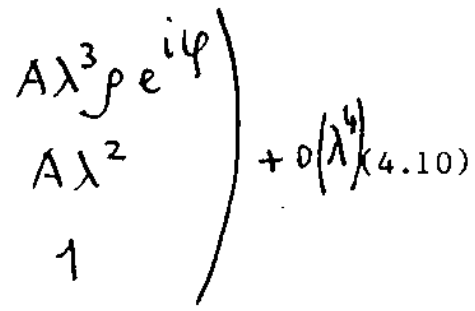

which will be used in the following.

We now consider the available experimental information on $A, \rho$ and $\phi$ $\left[\lambda\right.$ is given in Eq. (4.6)]. A is fixed by the b 1 ifetime $\tau_{B}$ and the semileptonic branching ratio $B_{S L}=B(b \rightarrow e v X)$. The semi-leptonic width $\Gamma_{S L}=B_{S L} / \tau_{B}$ can be computed by the parton model improved by QCD and phase space corrections. One could also add non-perturbative corrections to the spectator picture, typical of the parton model. These terms are model-dependent, but are small for the totally inclusive semi-leptonic width. One obtains:

$\tau_{B}\left|V_{b c}\right|^{2}=\frac{B_{S L} 10^{-13} s}{Z_{c}\left(1+\frac{\Gamma(b \rightarrow 4)}{\Gamma(b \rightarrow c)}\right)}=(2.9 \pm 0.6) 10^{-15} \mathrm{~s}$

where we used $B_{S L}=0.117 \pm 0.006^{18}$ and $Z_{c}=4.0 \pm 0.6$, the latter value 
being taken from Ref. 19. For $B^{0}-\bar{B}^{0}$ mixing only the combination $\tau_{B} A^{2}$ is important, which can directly be obtained from Eq. (4.11) $[\Gamma(b \rightarrow u) / \Gamma(b \rightarrow c)$ is smal1, see Eq. (4.15)]. Otherwise one can obtain A by using 20 $\tau_{B}=(1.11 \pm 0.16) 10^{-12 s:}$

$$
A=1.05 \pm 0.17
$$

Note that, since $A \sim 1,\left|v_{c b}\right| \sim\left|v_{t s}\right| \sim A \lambda^{2}$ indeed turn out to be of order $\lambda^{2}$.

$\rho$ is fixed by $R=\Gamma(b \rightarrow u) / \Gamma(b \rightarrow c)$ [where $\Gamma(b \rightarrow u)$ means $\Gamma(b \rightarrow u e v)$, i.e., the semi-leptonic width into charmless final states]:

$$
(0.47 \pm 0.02) R=\left|\frac{V_{u b}}{V_{c b}}\right|^{2}=(\lambda \rho)^{2}
$$

where the numerical factor is obtained from the parton model plus phase space and $Q C D$ corrections. $R$ is determined by the electron spectrum near the end-point. Clearly:

$$
\frac{d \Gamma}{d E_{e}}=\Gamma(b \rightarrow n)\left[\frac{1}{\Gamma(b \rightarrow u)} \frac{d \Gamma(b+u)}{d E_{e}}\right]+\Gamma(b \rightarrow c)\left[\frac{1}{\Gamma(b \rightarrow c)} \frac{d \Gamma \mid b \rightarrow c)}{d E_{e}}\right] \text { (4.14) }
$$

A priori there is some model dependence in the calculation of the normalized spectra. Various models have been tried 21 . A posteriori the differences are found smaller than one could expect. Quoting from $\operatorname{Ref} .21$ : "The mistaken impression has been given that spectator quark and valence quark models give substantially different results. As we shall see, this is not the case". At $90 \%$ c.1., one has ${ }^{22}$ :

$$
\begin{aligned}
R<0.13 & \text { (Crystal Ba11) } \\
<0.12 & \text { (ARGUS) } \\
<0.04 & \text { (CLEO) }
\end{aligned}
$$

1 obtained the last number from Table I of Ref. 23 (CLEO). The quoted limit comes from $R=(1.0 \pm 1.9) \%$ at $1.64 \sigma$. From the same table, one also sees that different models and procedures lead to comparable (and more restrictive) results. In conclusion, there could be model dependence in extracting $R$ from the spectrum (but apparently there is not). But I claim that, once $R$ is extracted from the spectrum, then there are no reasonable arguments for model-dependence in the relation Eq. (4.13) between $R$ and $\left|V_{b u}\right| /\left|V_{b}\right|$, that in fact everybody is always using outside this very particular discussion. It is true that Grinstein et al. 24 find $\sim 1$ instead of $0.47 \pm 0.02$ in Eq. (4.13) by trying to reconstruct the total $b \rightarrow u$ width from the sum of many exclusive channels estimated by modelling the relevant form factors. However, for the totally inclusive semi-leptonic widths $\Gamma(b \rightarrow u)$ and $\Gamma(b \rightarrow c)$, we have no reasons to cast doubts on the parton model, which works so well even for systems as 1 ight as the $\tau$ lepton (when only one hadronic current is involved and inclusive rates are concerned). Note that from Ref. 24 (see their Fig. 1 and 2), it appears that both the spectrum and the rate agree for the $b \rightarrow c$ semi-leptonic transition when computed in the parton model and by adding up the $\mathrm{D}^{\star}$ and $\mathrm{D}$ exclusive contribution. So, if the parton model is right for the $b \rightarrow c$ transition which is dominated by the $D^{*}$ and $D$ final states, why should we not a fortiori trust the parton model for the $b \rightarrow \mathrm{u}$ case, where a multitude of final states contribute and there is a lot of phase space? 
And indeed the authors of Ref. 24 explicitly state that they view the discrepancy by a factor of two between their model and the parton model in the $b \rightarrow u$ case as a measure of the error in the procedure of addition of exclusive channels (while the model could still be appropriate for the shape of the electron spectrum near the end-point).

In conclusion, the CLEO result $R<0.04$ implies $\left|V_{b u}\right| /\left|v_{b c}\right|<0.14$ or $\rho<0.6$ (we essentially agree with Ref. 21). However, to be conservative and let people do their choice, we shall, in the following, present results for $\left|v_{b u}\right| /\left|v_{b c}\right|<0.20$ or $\rho<0.9$.

Recently, ARGUS presented evidence for $\mathrm{v}_{\mathrm{bu}} \neq 0$ :

$$
\begin{aligned}
& B\left(B^{ \pm} \rightarrow P \bar{P} \pi^{ \pm}\right)=(3.7 \pm 1.3 \pm 1.4) 10^{-4} \\
& B\left(B^{\circ} \rightarrow P \bar{P} \pi^{+} \pi^{-}\right)=(6.0 \pm 2.0 \pm 2.2) 10^{-4}
\end{aligned}
$$

(a signal of $32.7 \pm 7.7$ events over the background). The connection with $\left|v_{b u}\right| /\left|v_{b c}\right|$ is really model-dependent in this case. ARGus indicates the 1 imit

$$
\left|\frac{v_{b u}}{v_{b c}}\right|>0.07 \text { or } \rho>0.3
$$

which we will tentatively assume in the following, where we shall use $0.9<\rho<0.3$.

On $\cos \phi$ we observe that:

$$
\left|\frac{V_{t d}}{V_{t s}}\right|^{2}=\lambda^{2}\left(1+\rho^{2}-2 \rho \cos \phi\right)
$$

Thus $\left|\mathrm{v}_{\mathrm{td}}\right|$ is maximum for $\cos \phi=-1$ :

$$
\left|\frac{v_{t d}}{V_{t s}}\right|<\lambda(1+\rho) \simeq \frac{1}{2.4}
$$

One might be interested in maximizing $\left|v_{t d}\right|$ in order to make the predicted amount of $B_{\mathrm{d}}^{0}-\overline{\mathrm{B}}_{\mathrm{d}}^{0}$ mixing as large as possible. But for $\cos \phi=-1$ all $\mathrm{CP}$ violating effects vanish. Thus a compromise is necessary. In Figs. 5 and 6 we show the allowed intervals of cos $\phi$, once the constraint provided by the measured value of $|\varepsilon|$ is imposed, for different values of $m_{t}, \rho, A$ and $B_{k}<1$. The additional constraint from $\varepsilon^{\prime} / \varepsilon$ given by Eq. (3.27), not included in Figs. 5,6, would only be barely visible at large values of $\mathrm{m}_{t}$.

The values obtained for the mixing angles already allow some important semi-qualitative statements.

1) For kaons, the dominant charm contribution to $\Delta M_{b o x}$ is proportional to $\left(\mathrm{V}_{\mathrm{cd}}^{\star} \mathrm{V}_{\mathrm{cs}}\right)^{2} \mathrm{~m}_{\mathrm{c}}^{2}$, while the top quark contribution is proportional to $\left(v_{t d}^{*} v_{t s}\right)^{2} m_{t}^{2}$. Since $\left|v_{c d}^{\star} v_{c s}\right| \simeq \lambda$, while $\left|v_{t d}^{*} v_{t s}\right| \simeq A^{2} \lambda^{5}\left|1-p e^{-i \phi}\right|$ the top term is negligible for all practical values of $m_{t}$ (i.e., for $m_{t} \lesssim$ $\left.\mathrm{m}_{\mathrm{c}} / \lambda^{4} \sim 500-600 \mathrm{GeV}\right)$.

2) Going back to Eqs. (4.5) [or by Eq. (5.4) plus the fact that $\mathrm{v}_{\mathrm{ud}}^{*} \mathrm{v}_{\mathrm{us}}$ is real], we see that

$$
\operatorname{Im}\left(V_{c d} V_{c s}^{*}\right)=-\operatorname{Im}\left(V_{t d} V_{t s}^{*}\right) \simeq A^{2} \lambda^{5} \rho \sin \phi
$$




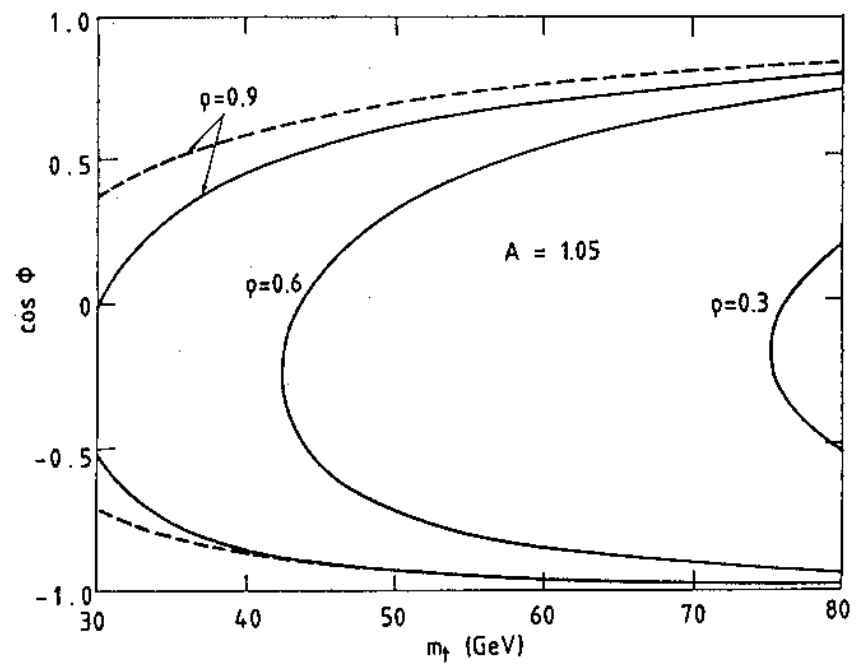

Fig. 5 Limits on $\cos \phi$ obtained from the experimental value of the CP violating parameter $|\varepsilon|$ for the kaon system, as functions of the top quark mass, $m_{t}$, for various values of $\rho$. The solid (dashed) lines include (do not include) the effect of the $\xi$ term. Here we have taken $A=1.05$ [the central value in Eq. (4.12)]. The parameters $\phi, \rho$ and $A$ are defined in $\mathrm{Eq} .(4.10)$. The indicates values of $\rho=0.9,0.6$ and 0.3 correspond to $R=\Gamma(b \rightarrow u) / \Gamma(b \rightarrow c)$ * $0.08,0.04$ and 0.009 respectively.

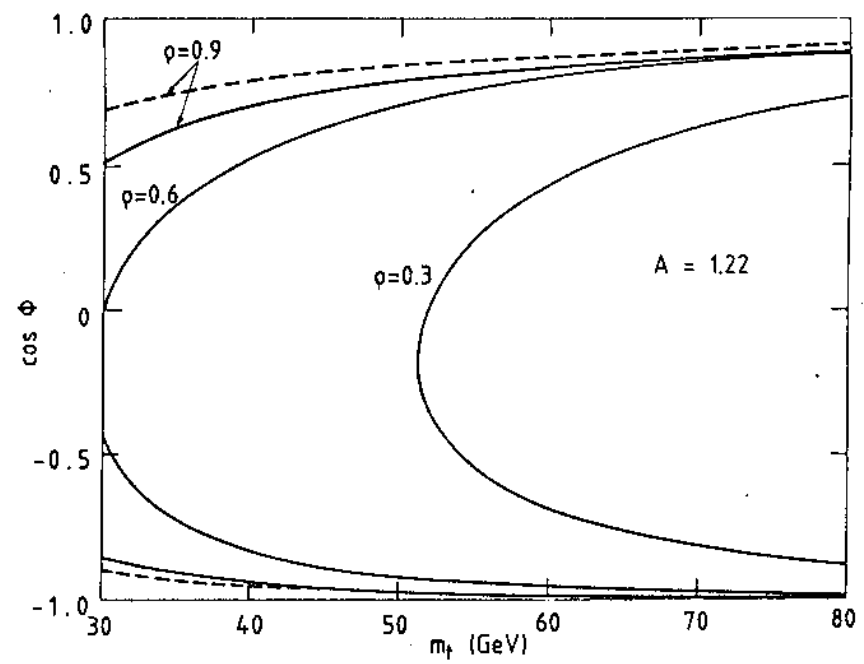

Fig. 6 Same as Fig. 5, but with $A=1.22$. 
As

$$
\operatorname{Im} z^{2}=2 \operatorname{Re} z \operatorname{Im} z
$$

then

$$
\frac{\operatorname{Im}\left(V_{t s} V_{t s}^{*}\right)^{2}}{\operatorname{Im}\left(V_{c d} V_{c s}^{*}\right)^{2}}=-\frac{\operatorname{Re}\left(V_{t d} V_{t s}^{*}\right)}{\operatorname{Re}\left(V_{c d} V_{c s}^{*}\right)} \sim-A^{2} \lambda^{4}(1-\rho \cos s)
$$

As a consequence the top quark contribution to $\varepsilon$ (i.e., to $\operatorname{Im} M_{12}$ ) is important as soon as $m_{t} 2 m_{c} / \lambda^{2} \sim 25 \div 30 \mathrm{Gev}$. Note that the short distance approximation is reasonably justified for $\varepsilon$ and not so much for $\Delta \mathrm{m}_{\mathrm{K}}$.

3) For charmed mesons $\left(D^{0}=c \vec{u}\right)$, the $D^{0}-\vec{D}^{0}$ mixing is predicted to be vexy small and cannot be reliably computed by the box diagram. In fact, the $b$ term is proportional to $\left(v_{c b} v_{y b}^{*}\right)^{2} m_{b}^{2}$, hence so small that the strange quark exchange of order of $\lambda^{2} m^{2}$ can still win. Typical order of magnitude estimates 1 ead to $(\Delta \mathrm{m} / \Gamma) \sim 10^{-2} \div 10^{-3}$. Experimentally 25 , at $90 \%$ c.1., $\mathrm{r}_{\mathrm{D}} \lesssim 1.4 \%$ (ARGUS), $\mathrm{r}_{\mathrm{D}} \lesssim 0.6 \%$ (TPS).

\section{CALCUlation OF THE BOX DiAgRAMS}

It is instructive to do the simple calculation of the box diagrams in Fig. 1. We shall explicitly consider the $B^{0}-\bar{B}^{0}$ system where we can restrict the calculation to the contribution of the top quark. The extension to the general case where two different heavy quarks are exchanged on the two sides of the box is left as an exercise.

of the two box diagrams in Fig. 1, it is sufficient to compute the first one. As we shall see, the second one $c$ an then be obtained for free. We shall do the computation in the unitary gauge where the $W$ propagator is $\left(-g^{\mu \nu}+k^{\mu} k^{\nu} / M_{W}^{2}\right) /\left(k^{2}-M_{W}^{2}\right)$ and there are no diagrams with unphysical scalars. In general, in a spontaneously broken gauge theory it is simpler and more rigorous to work in the so-called $\xi$-gauges. However, this particular problem is so simple and convergent that we can handle it directly. Thus, I do not have to invest time in explaining the $\xi-$ gauge methods.

It is convenient to neglect external quark momenta. All their components in the $B$ rest frame are of order $M_{B}$ and $c a n$ be ignored with respect to $M_{W}$ and $m_{t}$. If two quarks $i$ and $j$ are exchanged, the box amplitude from diagram a in Fig. I is proportional to (for me $\hat{k} \equiv k_{\mu} \gamma^{\mu}$ )

$$
\begin{aligned}
& \text { Box } \propto \int^{4} d^{4} \sum_{i, j} \bar{v}_{d} V_{i d}^{*} \gamma_{\mu} \frac{1-\gamma_{5}}{2} \frac{\hat{k}+m_{i}}{k^{2}-m_{i}^{2}} \gamma_{\nu} \frac{1-\gamma_{5}}{2} V_{i b} u_{b} . \\
& \cdot \bar{u}_{d} V_{j d}^{*} \gamma_{\sigma} \frac{1-\gamma_{5}}{2} \frac{\hat{k}^{2} m_{j}}{k^{2}-m_{j}^{2}} \gamma_{j} \frac{1-\gamma_{5}}{2} V_{j b} v_{b} \cdot\left(-g^{\mu \gamma^{\prime}}+\frac{k^{\mu} k^{\rho}}{M_{w}^{2}}\right) . \\
& \cdot\left(-g^{\nu \sigma}+\frac{k^{\nu}{ }^{\sigma}}{M_{w}^{2}}\right) \frac{1}{\left(k^{2}-M_{w}^{2}\right)^{2}}
\end{aligned}
$$

The quark masses in the numerator can be immediately dropped because they are killed by the chiral projector $\left(1-\gamma_{5}\right) / 2$. Denoting 


$$
\lambda_{i} \equiv V_{i b} V_{i d}^{*}
$$

we directly obtain (an overall factor is omitted)

$$
\begin{aligned}
& B_{0 x}=\sum_{i j} \lambda_{i} \lambda_{j} \bar{v}_{d} \gamma_{\mu} \gamma_{\tau} \gamma_{\nu} \frac{1-\gamma_{5}}{2} u_{b} \cdot \bar{u}_{d} \gamma_{\sigma} \gamma_{\lambda} \gamma_{\rho} \frac{1-\gamma_{s}}{2} v_{b} \\
& \cdot \int d^{4} k \frac{k^{\tau}\left(-g^{\mu} \rho_{+} \frac{k^{\mu} k^{\rho}}{M_{w}^{2}}\right)\left(-g^{\nu \sigma}+\frac{k^{\nu} k^{\sigma}}{M_{w}^{2}}\right) k^{\lambda}}{\left(k^{2}-m_{i}^{2}\right)\left(k^{2}-m_{j}^{2}\right)\left(k^{2}-M_{w}^{2}\right)^{2}}
\end{aligned}
$$

The integral is quadratically divergent. Fortunately the application of the GIM mechanism 12 directly leads to a convergent integral. In fact, note that

$$
\sum_{i} \lambda_{i}=0
$$

because the $V$ matrix is unitary and therefore $\Sigma_{i} \lambda_{i}=0$ [see Eq. (5.2)] being an off-diagonal element of the identity matrix. With an obvious notation, the box diagram is of the form Box $=\Sigma_{i, j} \lambda_{i} \lambda_{j} E\left(m_{i}, m_{j}\right)$. By using Eq. (5.3) we can then write:

$$
\begin{aligned}
\text { Box }=\sum_{i j} & \lambda_{i} \lambda_{j} E\left(m_{i}, m_{j}\right)=\sum_{i j} \lambda_{i} \lambda_{j}\left[E\left(m_{i}, m_{j}\right)-\right. \\
& \left.-E\left(0, m_{j}\right)-E\left(m_{i}, 0\right)+E(0,0)\right]
\end{aligned}
$$

This is now much more convergent. To see this in a simple way, we now restrict ourselves to the case of interest. The dominant top contribution is given by

$B_{0 x}=\lambda_{t}^{2}\left[E\left(m_{t}, m_{t}\right)-2 E\left(m_{t}, 0\right)+E(0,0)\right]$

where we used the fact, evident from Eq. (5.3), that $E\left(m_{t}, 0\right)=E\left(0, m_{t}\right)$. By observing that:

$$
\begin{aligned}
\frac{1}{\left(k^{2}-m^{2}\right)^{2}}+\frac{1}{k^{4}}-\frac{2}{k^{2}\left(k^{2}-m_{t}^{2}\right)}=\left(\frac{1}{k^{2}-m_{t}^{2}}-\frac{1}{k^{2}}\right)^{2}= \\
=\frac{m_{t}^{4}}{k^{4}\left(k^{2}-m_{t}^{2}\right)^{2}}
\end{aligned}
$$

we see that the quadratically divergent integral in Eq. (5.3) after the GIM treatment is reduced to the more sensible form:

$$
I=m_{t}^{4} \int d^{\nu} k \frac{k^{\top}\left(-g^{\mu \rho}+\frac{k^{\mu_{k}} \rho}{M_{w}^{2}}\right)\left(-g^{\nu \sigma}+\frac{k_{k}^{\nu}}{M_{w}^{2}}\right) k^{\lambda}}{k^{4}\left(k^{2}-m_{t}^{2}\right)^{2}\left(k^{2}-M_{w}^{2}\right)^{2}}
$$


Now there is a little bit of algebra to do with the Lorentz indices and the Dirac matrices. We take the numerator in the integral and we dot it into the fermion 1 ines:

$$
\begin{aligned}
& \gamma_{\mu} \gamma_{\tau} \gamma_{\nu} \frac{1-\gamma_{5}}{2} \otimes \gamma_{\sigma} \gamma_{\lambda} \gamma_{\rho} \frac{1-\gamma_{5}}{2}\left\{g^{\mu \rho} y^{\nu \sigma} k^{\tau} k^{\lambda}-g^{\mu \rho} \frac{k^{\top} k_{k}^{\nu} k^{\lambda}}{M_{W}^{2}}-\right. \\
& \left.-y^{\nu \sigma} \frac{k^{\top} k^{\mu}{ }_{k} \rho_{k} \lambda}{M_{W}^{2}}+\frac{k^{\tau} k^{\mu} k^{\rho} \mu_{k}^{\nu} k^{\sigma} k^{\lambda}}{M_{W}^{4}}\right\}
\end{aligned}
$$

where the symbol $\otimes$ separates the matrices from two different fermion lines. By using $\hat{k} \hat{k}=k^{2}, k^{\tau} \sigma^{\sigma}=1 / 4 g^{\mu \tau} k^{2}$ (because of symmetric integration in $d^{4} k$ ) and the identity:

$$
\begin{aligned}
& \gamma_{\mu} \gamma_{\tau} \gamma_{\nu} \frac{1-\gamma_{5}}{2} \otimes \gamma^{\nu} \gamma^{\tau} \gamma^{\mu} \frac{1-\gamma_{5}}{2}=\left(g_{\mu} \gamma_{\nu}-g_{\mu \nu} \gamma_{\tau}+\right. \\
& \left.+g_{\tau \nu} \gamma_{\mu}+i \epsilon_{\mu \tau \nu \rho} \gamma^{\rho}\right) \frac{1-\gamma_{s}}{2} \otimes\left(g^{\mu \tau} \gamma^{\nu}-g^{\mu \nu} \gamma^{\tau}+\right. \\
& \left.+g^{\tau \nu} \gamma^{\mu}-i \epsilon \mu \tau \nu \sigma \gamma_{\sigma}\right) \frac{1-\gamma_{5}}{2}=4 \gamma_{\mu} \frac{1-\gamma_{5}}{2} \otimes \gamma^{\mu} \frac{1-\gamma_{5}}{2}
\end{aligned}
$$

we finally obtain

$$
B o x=\lambda_{t}^{2} \vec{v}_{d} \gamma_{\mu} \frac{1-\gamma_{5}}{2} u_{b} \cdot \bar{u}_{d} \gamma^{\mu} \frac{1-\gamma_{5}}{2} v_{b}\left[I_{2}-\frac{2 I_{4}}{M_{W}^{2}}+\frac{I_{6}}{4 M_{w}^{4}}\right]
$$

where

$$
I_{2,4,6}=m_{t}^{4} \int d^{4} k \frac{k^{2}, k^{4}, k^{6}}{k^{4}\left(k^{2}-m_{t}^{2}\right)^{2}\left(k^{2}-M_{w}^{2}\right)^{2}}
$$

We can set the following identities and definitions:

$$
\begin{aligned}
& I_{2}=m_{t}^{4} \int d^{4} k \frac{1}{k^{2}\left(k^{2}-m_{t}^{2}\right)^{2}\left(k^{2}-m_{w}^{2}\right)^{2}}= \\
& =m_{t}^{4} \frac{\partial}{\partial m_{t}^{2}} \frac{\partial}{\partial M_{w}^{2}} \int d^{4} k \frac{1}{k^{2}\left(k^{2}-m_{t}^{2}\right)\left(k^{2}-M_{w}^{2}\right)}=m_{t}^{4} \frac{\partial}{\partial m_{t}^{2}} \frac{\partial}{\partial M_{w}^{2}} J^{(5.13)} \\
& I_{4}=m_{t}^{4} \int d^{4} k \frac{1}{\left(k^{2}-m_{t}^{2}\right)^{2}\left(k^{2}-M_{w}^{2}\right)^{2}}=m_{t}^{4} \frac{\partial}{\partial m_{t}^{2}} \int d^{4} \frac{1}{\left(k^{2}-m_{t}^{2}\right)\left(k^{2}-M_{w}^{2}\right)^{2}}= \\
& =m_{t}^{4} \frac{\partial}{\gamma m_{t}^{2}} J_{1}
\end{aligned}
$$


$-19-$

$$
I_{6}=m_{t}^{4} \int d^{4} k \frac{k^{2}-m_{t}^{2}+m_{t}^{2}}{\left(k^{2}-m_{t}^{2}\right)^{2}\left(k^{2}-m_{w}^{2}\right)^{2}}=m_{t}^{4} J_{1}+m_{t}^{2} I_{4}
$$

In order to compute $J$ and $J_{1}$ we use the famous identity:

$$
\begin{aligned}
& \int d^{4} k \frac{1}{\left(k^{2}-m_{1}^{2}\right)\left(k^{2}-m_{2}^{2}\right)\left(k^{2}-m_{3}^{2}\right)}= \\
& =2 \int d x d y d z \delta(1-x-y-z) \int d^{4} k \frac{1}{\left(k^{2}-m_{1}^{2} x-m_{2}^{2} y-m_{3}^{2} z\right)^{3}}
\end{aligned}
$$

Given the volume of the sphere of $x$ radius $R$ in $N$ dimensions

$$
V_{N}=\frac{\pi^{N / 2} R^{N}}{\Gamma(1+N / 2)}
$$

we obtain

$$
V_{4}=\pi^{2} R^{4} / 2 \rightarrow d V_{4}=\pi^{2} R^{2} d R^{2}
$$

By a wick rotation (which changes $k_{0}+i k_{0}$ ) $\int d^{4} k++i \int d^{4} k_{E}=+i \pi^{2} k_{E}^{2} d k_{E}^{2}$ and one finally obtains:

$$
\int \frac{d^{4} k}{\left(k^{2}-A^{2}\right)^{3}}=-i \pi^{2} \int \frac{k_{E}^{2} d k_{E}^{2}}{\left(k_{E}^{2}+A^{2}\right)^{3}}=\frac{-i \pi^{2}}{2 A^{2}}
$$

From these relations the following table of integrals can easily be derived:

$$
\begin{aligned}
& J=\frac{+i \pi^{2}}{M_{W}^{2}} \frac{\ln \eta}{1-\eta} \quad J_{1}=\frac{-i \pi^{2}}{M_{W}^{2}} \frac{1}{1-\eta}\left(1+\frac{\eta}{1-\eta} \ln \eta\right) \\
& I_{2}=\frac{-i \pi^{2}}{M_{W}^{2}} \frac{\eta}{(1-\eta)^{2}}\left(1+\eta+\frac{2 \eta}{1-\eta} \ln \eta\right) \\
& I_{4}=\frac{-i \pi^{2} \eta^{2}}{(1-\eta)^{2}}\left(2+\frac{1+\eta}{1-\eta} \ln \eta\right) \\
& I_{6}=\frac{-i \pi^{2} M_{w}^{2} \eta^{2}}{(1-\eta)^{2}}\left(1+\eta+\frac{2 \eta}{1-\eta} \ln \eta\right) \\
& \quad \eta=\frac{m_{t}^{2}}{M_{w}^{2}}
\end{aligned}
$$

Finally,

$$
\begin{aligned}
& I_{2}-\frac{2 I_{4}}{M_{W}^{2}}+\frac{I_{6}}{4 M_{W}^{4}}=\frac{i \pi^{2}}{M_{W}^{2}} \eta\left(\frac{1}{4}+\frac{9}{4} \frac{1}{1-\eta}-\frac{3}{2} \frac{1}{(1-\eta)^{2}}-\right. \\
& \left.-\frac{3}{2} \frac{\eta^{2} \ln \eta}{(1-\eta)^{3}}\right) \equiv \frac{i \pi^{2}}{M_{W}^{2}} A(\eta)
\end{aligned}
$$


By taking into account the overall factors left aside, the result can be cast into the form of an equivalent four fermion operator for the Hamiltonian:

$H_{\text {eff }} \simeq\left(\frac{g^{2}}{2}\right)^{2} \frac{\lambda_{t}^{2}}{16 \pi^{4}} \frac{\pi^{2}}{M_{w}^{2}} A(\eta) \frac{1}{2} \bar{d} \gamma_{\mu} \frac{1-\gamma_{5}}{2} b \bar{d} \gamma^{\mu} \frac{1-\gamma_{5}}{2} b$

$g$ is the $S U(2)$ weak coupling $\left(g^{2} / 8 M_{W}^{2}=G_{F} / \sqrt{2}\right),(2 \pi)^{-4}$ is the factor associated with the loop integration, a factor $-i$ was dropped because $S \sim-i H$ where $S$ is the $S-m a t r i x$ element, the spinors $\bar{v}_{d}, \ldots$, have been replaced by the corresponding operator fields $\mathrm{d}, \ldots$, and a factor of $\frac{1}{2}$ was added to compensate for exchange of the two identical $d \gamma_{\mu}\left(1-\gamma_{5}\right) / 2 b$ factors. Finally, we can write the result in the form:

$H_{\text {eff }}=\frac{G_{F}^{2}}{4 \pi^{2}} m_{t}^{2} \frac{A(\eta)}{\eta} \lambda_{t}^{2} \bar{d} \gamma_{\mu} \frac{1-\gamma_{5}}{2} b \bar{d} \gamma^{\mu} \frac{1-\gamma_{5}}{2} b$

Once the effective Hamiltonian has been written in operator form, there is no need of computing the second diagram. Note that, in fact, in the 1 imit of vanishing external momenta the loop integrals are identical in the two diagrams. The contribution of the second diagram corresponds to a different contraction of the fields in the Hamiltonian with the external quarks. We will take both contributions into account when taking the matrix element $\left\langle B_{0}\left|H_{\text {eff }}\right| \bar{B}^{0}\right\rangle$.

We now compute the above matrix element in the vacuum saturation (or valence) approximation. When the matrix element of a V-A current is taken between the vacuum and a pseudoscalar meson, only the axial current contributes (one cannot make a pseudovector out of only one momentum $P_{\mu}$ ). For $\pi \rightarrow \mu \nu$ one defines:

$$
\left\langle 0\left|\bar{u} \gamma_{\mu} \gamma_{s} d\right| \pi\right\rangle=\frac{i p_{\mu} f_{\pi}}{\sqrt{2 E_{p}}}
$$

With this normalization, experimentally $\mathrm{f}_{\pi} \approx 130 \mathrm{MeV}$ (similarly $\left.\mathrm{f}_{\mathrm{K}} \approx 160 \mathrm{MeV}\right)$. Thus, by restricting the sum over a complete set of intermediate states to the vacuum only, one obtains in the $B^{0}$ rest frame:

$$
\left\langle B^{0}\left|f_{\mu} j^{\mu}\right| \bar{B}^{\circ}\right\rangle=2 \cdot \frac{1}{4}\left(1+\frac{1}{3}\right) \frac{1}{2 m_{B}} m_{B}^{2} f_{B}^{2}
$$

We now explain one by one the factors which appear in the last expression. The factor of two is there because we can choose in two ways the current on the $\mathrm{B}^{0}$ side (this factor compensates the factor of $\frac{1}{2}$ that we had inserted in the Hamiltonian). The factor of $1 / 4$ is there because each current contains the projector $\left(1-\gamma_{5}\right) / 2$. The factor $4 / 3$ arises because the vacuum state can be inserted in two ways. This corresponds to the two original diagrams $a$ and $b$ in Fig. 1 which differ by the exchange of the two $b$ fields. A $(V-A) \otimes(V-A)$ product of currents is invariant under fierz rearrangement for colourless quarks. For coloured quarks the colour indices have also to be rearranged and one obtains: (see Eq. $(3.3)$ )

$$
\begin{aligned}
& \bar{J}_{1 L} \gamma_{\mu} b_{1 L} J_{2 L} \gamma^{\mu} b_{2 L}=\frac{1}{3} J_{1 L} \gamma_{\mu} b_{2 L} \bar{d}_{2 L} \gamma^{\mu} b_{1 L}+ \\
& +2 \sum_{A} \bar{d}_{1 L} \gamma_{\mu} \lambda^{A} b_{2 L} \bar{d}_{2 L} \gamma^{\mu} \lambda^{A} b_{1 L}
\end{aligned}
$$

where $\lambda_{i j}^{A}$ are the $3 \times 3$ colour generator matrices with normalization 
$\operatorname{Tr} \lambda^{A} \lambda^{B}=\frac{1}{2} \delta^{A B}$ [to derive Eq. $(5.27)$ is a nice exercise for you to do]. The octet-octet term cannot contribute when each octet is sandwiched between the vacuurn and the colour singlet $B$ state. Thus the two contractions differing by $b_{1}$ - $b_{2}$ would contribute $(1+1)=2$ for colourless quarks and $(1+1 / 3)=4 / 3$ for triplet coloured quarks. Thus for coloured quarks we set

$$
\left\langle B^{0}\left|\left(\bar{d} \gamma_{\mu} \frac{1-\gamma_{5}}{2} b\right)^{2}\right| \bar{B}^{0}\right\rangle=\frac{1}{3} B_{B} f_{B}^{2} m_{B}^{2}
$$

where $B_{B}$ is a factor which is inserted to take all possible deviations from the vacuum saturation approximation into account. Finally, we have

$$
M_{12}=\frac{G_{F}^{2} m_{t}^{2}}{12 \pi^{2}} B_{B} f_{B}^{2} m_{B} \lambda_{t}^{2} \frac{A(\eta)}{\eta}
$$

$\mathrm{A}(\eta) / \eta$ is a slowly decreasing monotonic function of $\eta$, plotted in Fig. 7, which is 1 at $\eta=0,3 / 4$ at $\eta=1$ and $1 / 4$ at $\eta \rightarrow \infty$.

For $B$ mesons the top contribution is by far the only one that matters. For kaons the charm contribution, similar to Eq. $(5,29)$, is sufficient to Re $M_{12}$, while for $\operatorname{Im~} M_{12}$ (which determines $\varepsilon$ ) one has also to compute the term with $c$ and $t$ quarks on the two sides of the box. The calculation is entirely analogous to the one performed here and the result can be found in the literature 26 .

\section{QCD CORRECTIONS TO BOX DIAGRAMS}

The box diagram calculation of the last section is based on neglecting the strong interactions at short distances, which is valid if both $\mathrm{M}_{W}$ and the exchanged quark masses are large. The QCD corrections to the strong interaction-free result can be simply obtained in terms of the anomalous dimensions of the relevant operators in the short distance expansion. We shall now derive the result for $m_{t}<M_{W}$ and then comment on the case that $m_{t}>M_{W}$.

We start again from the general discussion in Section 2 of the twostep operator expansion. For $\mathrm{M}_{\mathrm{W}} \gg \mathrm{m}_{t}$ the $\mathrm{W}$ propagators in Eq. (3.1) are only different from zero at short distances, so that the operator expansion for the product of two weak charged currents can be used 27 (including the effects of strong interactions):

$$
J_{\mu}(z) J^{\mu}(0) \simeq c_{+} O_{+}(0)+c_{-} O_{-}(0)+0\left(\frac{1}{M_{W}^{2}}\right)
$$

where [recall Eq. (3.3)]

$$
\begin{aligned}
O_{ \pm} & =\frac{1}{2}\left(\bar{d}_{L} \gamma_{\mu} t_{L} \bar{t}_{L} \gamma^{\mu} b_{L} \pm d_{L} \gamma_{\mu} b_{L} \bar{t}_{L} \gamma^{\mu} t_{L}\right)= \\
= & \frac{1}{2}\left\{\left(1 \pm \frac{1}{3}\right) \bar{d}_{L} \gamma_{\mu} t_{L} \bar{t}_{L} \gamma^{\mu} b_{L} \pm\right. \\
& \left. \pm 2 \sum_{A} \bar{d}_{L} \gamma_{\mu} \lambda^{A} t_{L} \bar{t}_{L} \gamma^{\mu} \lambda^{A} b_{L}\right\}
\end{aligned}
$$

The second equality was obtained by Fierz rearrangement. $C_{ \pm}$are computable coefficients we 11 known 27 from the physics of weak non-leptonic decays. At the leading logarithmic level: 


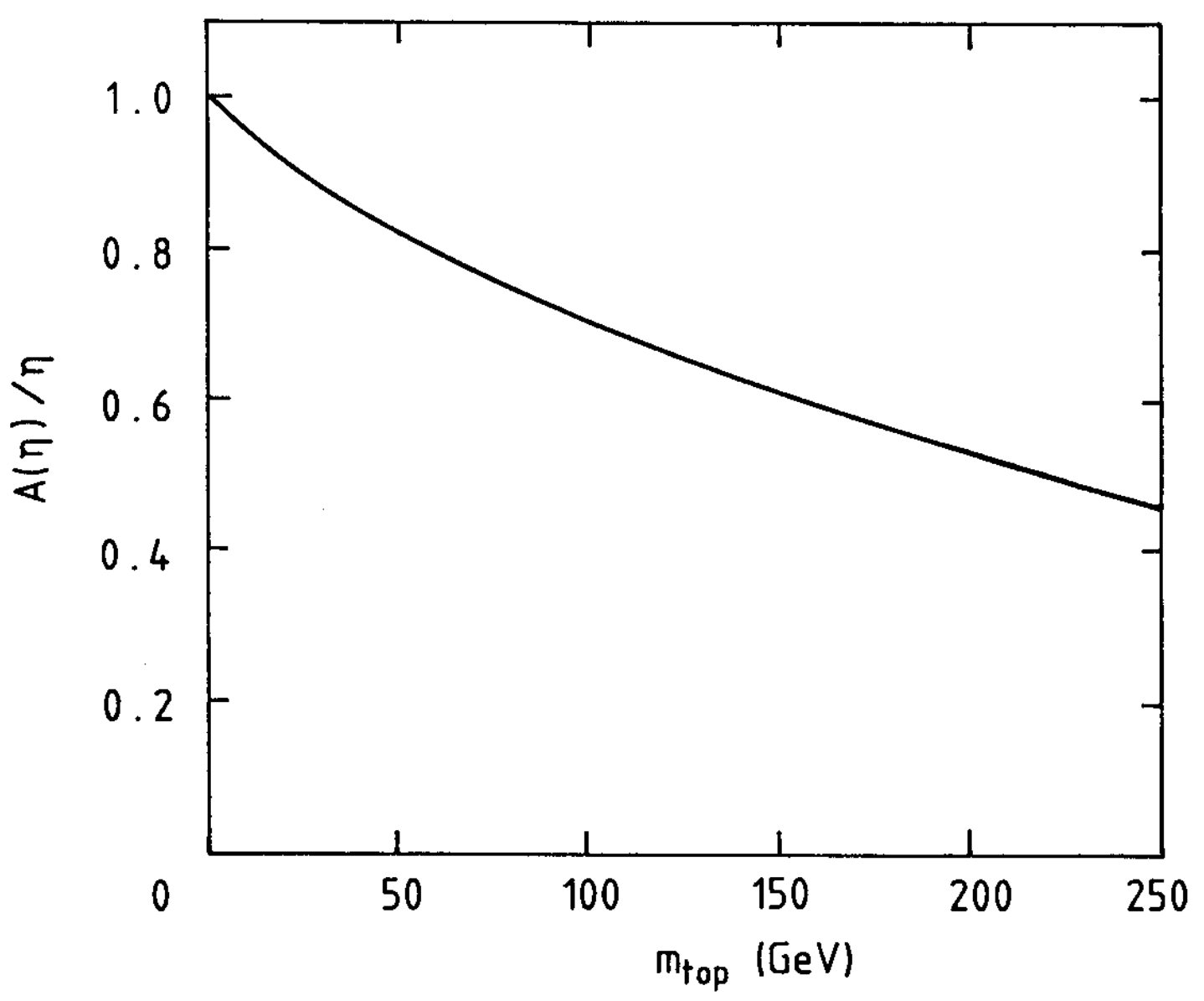

Fig. 7 The function $A(\eta) / \eta\left(\eta=m_{t}^{2} / M_{W}^{2}\right)$ defined in Eq. (5.22). 


$$
\begin{aligned}
& c_{ \pm}=\left[\frac{\alpha_{s}(\mu)}{\alpha_{s}\left(M_{w}\right)}\right]^{d \pm} \\
& b_{0}(f)=11-\frac{2}{3} f
\end{aligned} \quad\left\{\begin{array}{l}
d-=\frac{4}{b_{0}(f)} \\
d_{+}=\frac{-2}{b_{0}(f)}
\end{array}\right.
$$

where $\alpha_{s}$ is the QCD running coupling and $f$ is the number of excited flavours between $\mu$ and $M_{W}$ (it can vary along the road, but we forget that for a moment). In the first step, when we shrink the $W 1$ ines to a point we get:

$$
\mathrm{JJJJ} \rightarrow \mathrm{C}_{+}^{2} \mathrm{O}_{+} \mathrm{O}_{+}+2 \mathrm{C}_{+} \mathrm{C}_{-} \mathrm{O}_{+} \mathrm{O}_{-}+\mathrm{C}_{-}^{2} \mathrm{O}_{-} \mathrm{O}_{-}
$$

Since $f=6$ between $m_{t}$ and $M_{W}$, we set $\mu=m_{t}$ and obtain $\left(b_{0}(6)=7\right)$ :

$$
c_{+}^{2} \Rightarrow 2 d_{+}=-\frac{4}{7}, c_{+} c_{-} \Rightarrow d_{+}+d_{-}=\frac{2}{7}, c_{-}^{2} \Rightarrow 2 d_{-}=\frac{8}{7} \text { (6.5) }
$$

In the subsequent steps, we shrink the top quark lines to a point. We end up with a four-fermion operator of the form $d_{L} \gamma_{\mu} b_{L} d_{L} \gamma^{\mu} b_{L}$ or $\Sigma_{A} d_{L} \gamma_{1} \lambda^{A_{b}} d_{L} d_{L} \gamma^{\mu} \lambda^{A_{b}} b_{L}$ which can always be expressed in terms of $0_{ \pm}$[the symmetric and antisymmetric combinations completely analogous to $\mathrm{Eq} .(6.2)]$. When finally the matrix element is taken, $0_{-}$does not contribute because the two modes of vacuum insertion cancel each other. Thus when going from $m_{t}$ down to $m_{b}$, the relevant coefficient is $c_{+}$(with $f=5$, i.e., $\left.b_{0}(5)=23 / 3\right)$. Up to now we have obtained the result

$\left.\eta_{Q C D} \simeq \frac{\alpha_{s}\left(m_{b}\right)}{\alpha_{s}\left(m_{t}\right)}\right]^{\frac{-6}{23}}\left\{a\left[\frac{\alpha_{s}\left(m_{t}\right)}{\alpha_{s}\left(M_{w}\right)}\right]^{-\frac{4}{7}}+b\left[\frac{\alpha_{s}\left(m_{t}\right)}{\alpha_{s}\left(M_{w}\right)}\right]^{\frac{2}{7}}+c\left[\frac{\alpha_{s}\left(m_{t}\right)}{\alpha_{s}\left(M_{w}\right)}\right]^{\frac{8}{7}}\right\}$

but the coefficients $a, b$ and $c$ remain to be determined. Of course, $a+b+c=1$ because the curly bracket must approach 1 for $m_{t} \rightarrow M_{W}$ (no correction while running from $M_{W}$ down to $m_{t}$ if there is no distance!). $a, b$ and $c$ are obtained by colour traces. Before the top quark contractions we have (all indices are colour indices):

$H \sim \bar{q}_{a} C_{a b} t_{b} \bar{t}_{c} C_{c d} q_{d} \bar{q}_{s} D_{s t} t_{t} \bar{t}_{u} D_{u r} q_{v}$

Here $\mathrm{C}_{a b} \mathrm{C}_{c d}\left(\mathrm{D}_{s t} \mathrm{D}_{\mathrm{uv}}\right)$ are the colour matrices in the two currents previously joined by a $W$ line:

$$
C_{a b} C_{c d}=C \otimes C=A\|\otimes\|+B \sum_{A} \lambda^{A} \otimes \lambda^{A}
$$

$C$ (or D) can correspond either to $0_{+}$or to $0_{-}$. If $C \sim 0_{+}$then [see $\mathrm{Eq} .(6.2)] \mathrm{A}=4 / 3, \mathrm{~B}=2$ while. for $\mathrm{C} \sim 0_{-}$then $\mathrm{A}=2 / 3, \mathrm{~B}=-2$. We now contract $t_{b}$ with $\bar{t}_{u}$ (giving $\delta_{b u}$ ) and $\bar{t}_{c}$ with $t_{t}\left(\delta_{c t}\right)$ so that

$$
H \sim \bar{q}_{a}(C D)_{a v} q_{v} \bar{q}_{s}(D C)_{s d} q_{\alpha}
$$

Finally, we make the two possible projections over colour singlets by multiplying by either $\delta_{a v} \delta_{s d}$ or $\delta_{a d} \delta_{v s}$ which leads to TrCDTrDC $+\operatorname{TrCDDC}$. If we denote by $A^{\prime}$ and $B$ ' the analogous coefficients for $D D$ of $A$ and $\mathrm{B}$ for $\mathrm{C} \otimes \mathrm{C}$ [defined in $\mathrm{Eq} .(6.8)$ ] we obtain: 
$M_{12} \simeq 12 A A^{\prime}+4\left(B A^{\prime}+A B^{\prime}\right)+\frac{22}{3} B B^{\prime}$

This leads to:

$O_{+} O_{+}: O_{+} O_{-}+O_{-} O_{+}: 0_{-} O_{-}=\frac{3}{2}:-1: \frac{1}{2}$

Thus with the right normalization:

$\eta_{Q C D}=\left[\frac{\alpha_{s}\left(m_{b}\right)}{\alpha_{s}\left(m_{t}\right)}\right]^{-\frac{6}{23}}\left\{\frac{3}{2}\left[\frac{\alpha_{s}\left(m_{t}\right)}{\alpha_{s}\left(M_{W}\right)}\right]^{-\frac{4}{7}}-\left[\frac{\alpha_{s}\left(m_{t}\right)}{\alpha_{s}\left(M_{W}\right)}\right]^{\frac{2}{7}}+\frac{1}{2}\left[\frac{\alpha_{s}\left(m_{t}\right)}{\alpha_{s}\left(M_{W}\right)}\right\}^{\frac{8}{7}}\{(6.12)\right.$

This formula has been derived for $m_{b} \ll m_{t} \ll M_{W}$. Strictly speaking, perturbative QCD with a given number of massless flavours can only be applied far away from quark thresholds. In the regions across the thresholds we can only guess reasonable extrapolations. Note that in Eq. (6.12) for $\eta_{\text {. }}, \alpha$ runs with five flavours in the first factor (between $m_{b}$ and $m_{t}$ ) and with six flavours in the second factor (between $m_{t}$ and $\left.M_{W}\right)$. For example, we can impose that $\alpha_{s}$ is continuous in the two regions by setting

$$
\begin{aligned}
& \alpha_{s}^{f=5}\left(Q^{2}\right)=4 \pi\left[b_{0}(5) \ln \frac{Q^{2}}{\Lambda^{2}}\right]^{-1} \\
& \alpha_{s}^{f=6}\left(Q^{2}\right)=4 \pi\left[b_{0}(b) \ln \frac{Q^{2}}{\Lambda^{2}}+c\right]^{-1}
\end{aligned}
$$

where $C$ is fixed by $\alpha_{S=5}^{f=5}\left(m_{t}\right)=\alpha_{S}^{f=6}\left(m_{t}\right)$. This amounts to change $\Lambda$ for each $f$. We put $C$ in $\alpha_{s} f=6$ so that our $\Lambda$ is $\Lambda$ (leading order) for $f=5$ which is what we can measure below the top threshold.

When $m_{t}$ increases up to $M_{W}$ and beyond (not too much beyond because we know that $m_{t} \lesssim 2 \div 3 \mathrm{M}_{\mathrm{W}}$ ), the interplay between the logarithmic terms for the anomalous dimensions and the mass terms [the function $A(\eta) / \eta$ in Eq. (5.29)] becomes more complicated. The logs resumed by $\eta_{\text {ocD }}$ are still there. The expression for $\eta_{\mathrm{OCD}}$ will change a bit but not much (for example, the running will all the way occur with $f=5$ ). However, the function $A(\eta) / \eta$ could be deformed by additional QCD corrections because $\eta_{O C D}$ is the right multiplying factor in the limit $\eta \rightarrow 0$. However, since $A(\eta) / \eta$ is a slowly varying function and $\eta_{Q C D}$ is almost a constant ( $\eta_{Q C D} \sim$ $0.80-0.85$ ) for $m_{t} \approx 40 \div 200 \mathrm{GeV}$, it is presumably correct to use the product $\eta_{Q C D}(A(\eta) / \eta)$ for the whole physically interesting range of $m_{t}$.

7. $B^{0}-\bar{B}^{0}$ MIXING IN THE STANDARD MODEL

By collecting Eqs. (3.9) and (5.29), we finally obtain the top exchange contribution to $\Delta \mathrm{m} / \Gamma$ for a $B_{q}$ meson $(q=d, s)$ : 
$x_{q} \equiv\left(\frac{\Delta m}{\Gamma}\right)_{B_{q}}=\frac{G_{F}^{2}}{6 \pi^{2}} m_{t}^{2} \tau_{B_{q}} B_{B_{q}} f_{B_{q}}^{2} m_{B_{q}}\left|V_{t b} V_{t q}^{*}\right|^{2} \frac{A(\eta)}{\eta} \eta_{Q C D}(7.1)$

where $A(\eta) / \eta$ and $\eta_{O C D}$ are given in Eqs. (5.22) and (6.12) respectively. Recall that [Eq. ( 3.5$)] x_{q}=x_{q}^{2} /\left(2+x_{q}^{2}\right)$. The first thing to observe is that the ratio $x_{d} / x_{s}$ can be predicted with little ambiguity. From Eq. (7.1), one in fact obtains:

$$
\frac{x_{d}}{x_{s}}=\left|\frac{v_{t d}}{v_{t s}}\right|^{2}\left(1+5 u(3)_{f l} \text { breaking }\right)
$$

where the $d$ - s symmetry breaking correction arises from all quantities which in Eq. (7.1) carry a q label, apart from the mixing angles which are explicitly factored out. Although the corrective term may well be sizeable (and is probably negative), it is safe to state that the main factor in Eq. (7.2) is the ratio $\left|v_{t d} / v_{t s}\right|^{2} \leqslant 1 / 5$. Namely $x_{d}$ is Cabibbo suppressed with respect to $x_{s}$ (in fact, the present bound roughly corresponds to $\left.\left|v_{t d}\right| \leqslant 2 \theta_{c}\left|v_{t s}\right|\right)^{s}$. This is why $r_{d}$ was expected to be small. Then from the experimental value of $r_{d}$, one immediately deduces that $r_{s}$ must be near one. Precisely $x_{d}>0.13$ implies $r_{s} 20.75-0.80$ depending on the precise input maximum value of $\left|v_{t d} / v_{t s}\right|$. The resulting allowed region in the $r_{d}, r_{s} p l a n e$ is plotted in Fig. 8 together with the ayailable experimental information. One sees that the MARK II 1 imits 28 are potentially dangerous for the Standard Model with three families. However, the interpretation of the MARK II results in terms of $r_{d}, r_{s}$ requires an assumption on the probabilities $P_{d}$, $P_{s}$ that the produced $b$ quark picks up a $\vec{d}$ or $\vec{s}$ companion. For example the allowed region for the Standard Model with three families is not much restricted by the MARK I result if $\mathrm{P}_{\mathrm{d}} \simeq 0.35, \mathrm{P}_{\mathrm{s}} \simeq 0.10$ (which, however, leaves a $\mathrm{r}$ ather generous $20 \%$ for $b \bar{c}$ and b-carrying baryons).

The value of $x_{d}$ is more uncertain. One can write the approximate expressions valid for $\mathrm{m}_{\mathrm{t}} \lesssim \mathrm{M}_{\mathrm{W}}$ :

$$
x_{d} \simeq 0.15\left[\frac{\tau_{B}\left|V_{t d}\right|^{2}}{3.310^{-16} \mathrm{~s}}\right]\left[\frac{B_{B_{d}} f_{B d}^{2}}{(0.14 \mathrm{GeV})^{2}}\right]\left[\frac{m_{t}}{40 \mathrm{GeV}}\right]^{2}
$$

$$
X_{d}=(0.26 \pm 0.05) \frac{1}{4}\left(1+\rho^{2}-2 \rho \cos \phi\right)\left[\frac{B_{B_{d}} f_{B_{\alpha}}^{2}}{(0.14 \mathrm{GeV})^{2}}\right]\left[\frac{m_{t}}{40 \mathrm{GeV}}\right]^{2}(7.4)
$$

Recall that experimentally ARGUS finds: $x_{d}=0.73 \pm 0.18$. The main unknowns are $\tau_{B}\left|v_{t d}\right|^{2}, B^{\frac{1}{2}} f_{B}$ and $m_{t}$. The errors on $\tau_{B}$ and $\left|v_{t d}\right|$ are related so that the two factors should be kept together. $\left|v_{\text {, }}\right|$ is maximum for an extremal value of the Kobayashi-Maskawa phase. However, at extremal values of the phase all CP violation effects vanish. The experimental values of $\varepsilon$ and $\varepsilon^{\prime / \varepsilon}$ therefore impose some constraints on how large $\mid v_{t d} l$ can be. Only mild restrictions are found (which become weaker with increasing $m_{t}$ ) if one takes $B_{K} \lesssim 1$ and $\left|v_{b u} / v_{b c}\right| \lesssim 0.2$ (see 


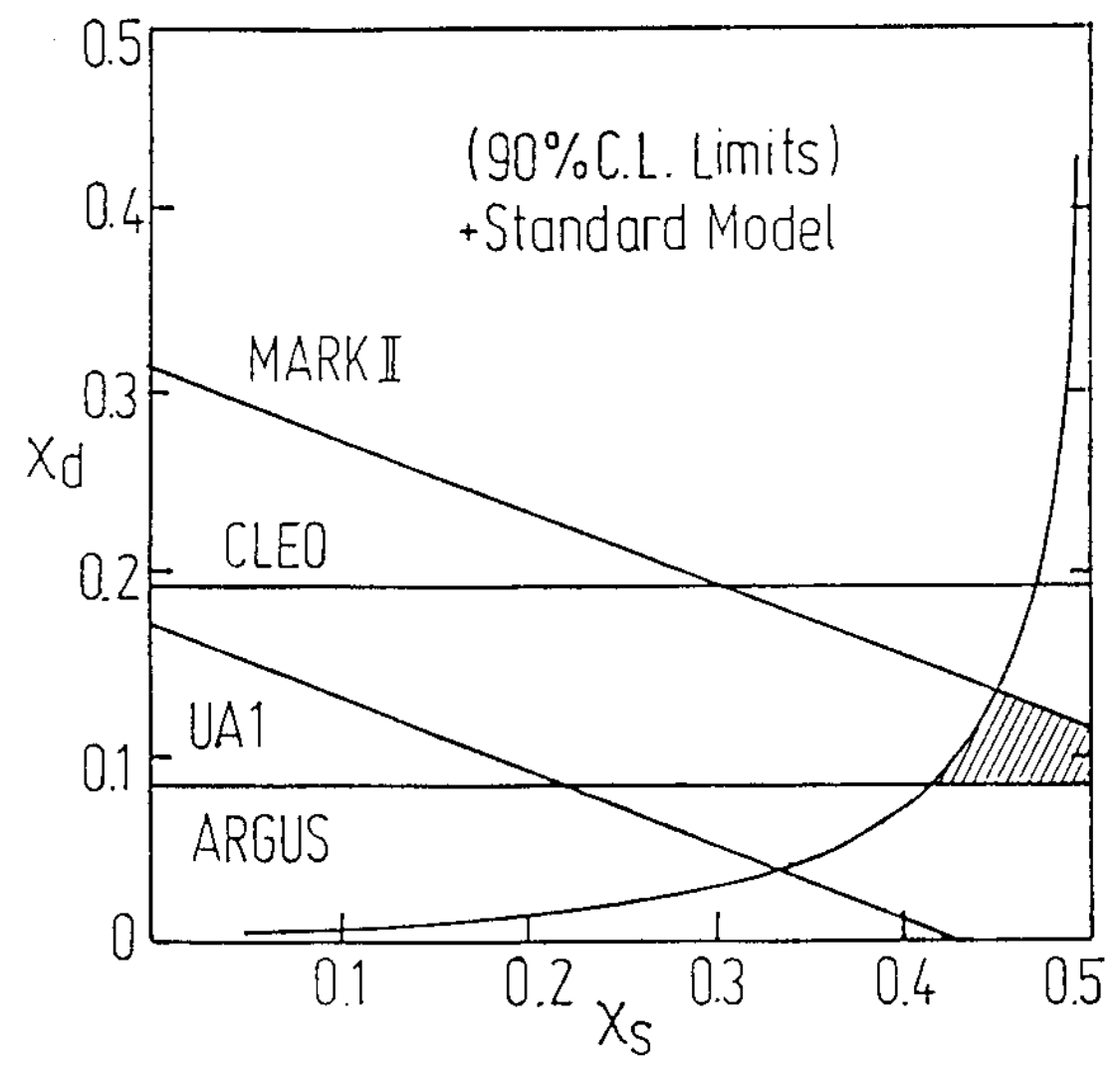

Fig. 8 The experimentally allowed region 10 in the $\chi_{\mathrm{d}}-\chi_{\mathrm{s}}$ plane ( $\chi$ is defined in Eq. (2.19) and the CKM model constraint. The shaded area is the region allowed by the present experiments and the CKM model. 
Figs $; 5$ and 6$)$. With these assumptions one finds for $\tau_{B}\left|v_{t d}\right|^{2}$ the
range

$$
\tau_{B}\left|V_{t d}\right|^{2} \simeq(0.02 \div 6.2) 10^{-16} \mathrm{r}
$$

The factor $B_{B} f^{2}$ contains all the uncertainties arising from the hadronic matrix element. The parameter $B$ should approach one when the mass of the meson increases. In fact, the vacuum saturation approximation should be better when gluon emission from the quark legs is inhibited by large transferred momenta of order the meson mass (Zweig rule). This trend appears to be supported by some recent lattice evaluations of $\mathrm{B}_{\mathrm{P}}$ and $\mathrm{B}_{\mathrm{B}} 29,30$. The corresponding results are closer to unity than those obtained for $B_{K}$ by several methods. The most reliable results on $B$ are collected in Table 129,33 . Similarly the pseudo-scalar decay constants $\mathrm{f}_{\mathrm{K}}, \mathrm{f}_{\mathrm{D}}, \mathrm{f}_{\mathrm{B}} \mathrm{can}$ be estimated mainly by QCD sum rules and lattice calculations. The available results are collected in Table $2^{34,40}$. The tentative conclusion that was derived in Ref. 7 is given by:

$$
B_{B_{d}}^{1 / 2} f_{B d}=(140 \pm 40) M e V
$$

The implications on $m_{t}$ are illustrated in Figs. 9 and $10^{7}$. One obtains that almost certainly $m_{t}>45 \mathrm{GeV}$. The most likely range for $m_{t}$ is given by $90 \mathrm{GeV}<\mathrm{m}_{\mathrm{t}}<150 \mathrm{GeV}$.

In conclusion the ARGUS result $c$ an be accommodated in the standard Model with three families provided that $m_{t}$ is large. The first important experimental check is to measure the mixing parameter $r_{\text {s }}$ for the ${ }^{B}$ mesons. If $r_{s}$ is not nearly maximal, the most obvious possibility is a fourth family.

It is interesting to remark that in the presence of a fourth generation, because of the more relaxed unitarity constraints on the CKM matrix elements, even the top quark contribution by itself (i.e., without invoking the additional terms from the new up-type quark $t^{\prime}$ ) can lead to drastically different values of both the $x$ atio $x_{d} / x_{s}$ and the quantity $x_{d}$. For example, the analysis of mixing angles in Ref. 41 leads to $v_{\text {td }} \lesssim$ 0.017 [note that $\mathrm{Eq}$. (7.5) corresponds to $\left.\mathrm{v}_{\mathrm{td}} \lesssim 0.026\right)$ for three generations. For four generations, their bound on $v_{t d}$ is relaxed by almost one order of magnitude - $v_{t d} \lesssim 0.15-$ which implies that practically all values of $r_{d}$ are possible.

Before closing this section, it is important to recall that for kaons the short distance contribution to $\Delta \mathrm{m}_{\mathrm{K}}$ (which essentially arises from $c$ quark exchange) is not sufficient to explain the observed value if $\mathrm{B}_{\mathrm{K}} \lesssim 1$. With very good approximation:

$$
\frac{\Delta m_{k}(\text { box })}{\Delta m_{k}(\text { exp })}=0.7\left[\frac{m_{c}}{1.5 G_{e V}}\right]^{2} B_{k}
$$

clearly, the range of relevant momenta between $m_{K}$ and $m_{c}$ is too low for a neglect of long distance effects. The situation is different for Im $M_{12}$, i.e., for $\varepsilon$. The CKM phases are only present in the heavy quark sector. The t quark exchange is also important. Thus in this case the range of relevant virtual momenta is between $m_{c}$ and $m_{t}$ and the dominance of short range effects is more plausible. 
TABLE 1

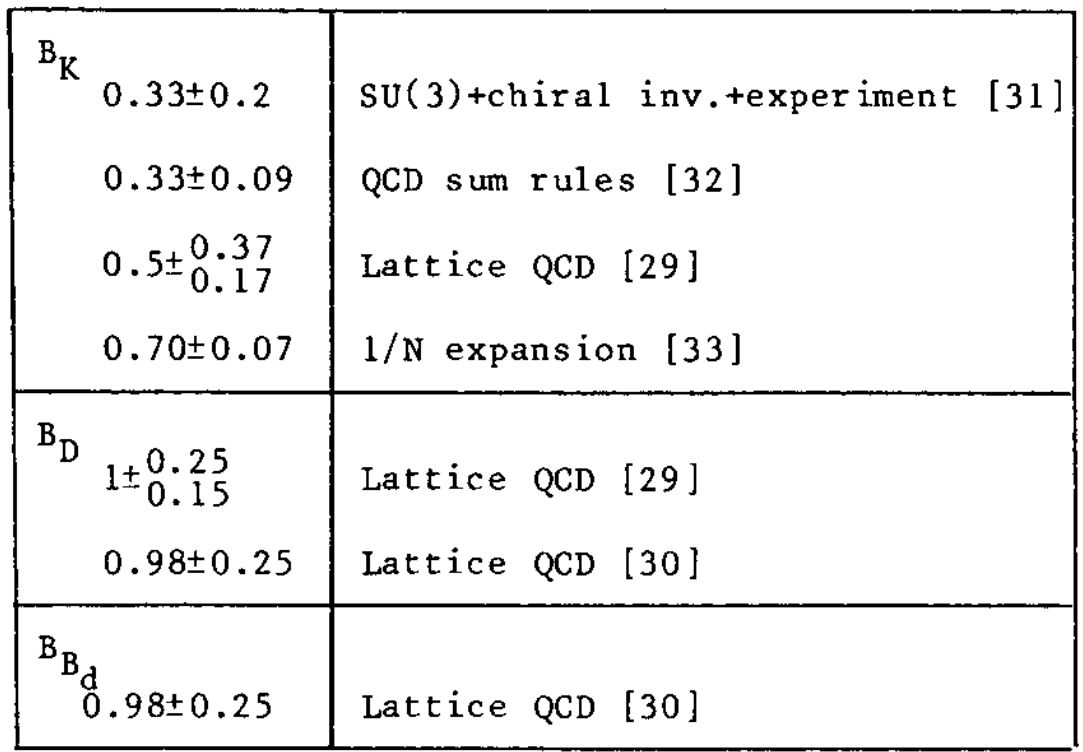

TABLE 2

$f_{\pi}=130 \mathrm{MeV}, f_{K}=160 \mathrm{MeV}$

\begin{tabular}{|c|c|c|}
\hline $\mathrm{f}_{\mathrm{D}}$ & & \\
\hline $\begin{array}{l}\sim 220(\mathrm{MeV}) \\
\sim 165 \\
\sim 170 \\
170 \pm 20 \\
220 \pm 25 \\
180 \pm 25 \\
128 \pm 25 \\
<290\end{array}$ & $\begin{array}{c}\text { QCD sum rules, } \\
" \\
" \\
" \\
\text { Lattice QCD, } \\
" \text { " } \\
\text { Exp. MARK III, }\end{array}$ & $\begin{array}{ll}\operatorname{Ref} .[34] \\
\operatorname{Re} . & {[35]} \\
\operatorname{Ref} . & {[36]} \\
\operatorname{Re} f . & {[37]} \\
\operatorname{Ref} . & {[38]} \\
\operatorname{Ref} . & {[29]} \\
\operatorname{Ref.}[ & {[30]} \\
\operatorname{Ref} . & {[39]}\end{array}$ \\
\hline \multicolumn{3}{|l|}{$\mathrm{f}_{\mathrm{B}}$} \\
\hline $\begin{array}{l}\sim 140 \\
\sim 95 \\
\sim 130 \\
190 \pm 30 \\
180 \pm 20 \\
175 \pm 30\end{array}$ & $\begin{array}{c}\text { QCD sum rules, } \\
" 1 \\
" \\
" \\
"\end{array}$ & $\begin{array}{ll}\operatorname{Ref} .[34] \\
\operatorname{Re} . & {[35]} \\
\operatorname{Re} f . & {[36]} \\
\operatorname{Re} f . & {[40]} \\
\operatorname{Ref} . & {[37]} \\
\operatorname{Ref.} & {[38]}\end{array}$ \\
\hline
\end{tabular}




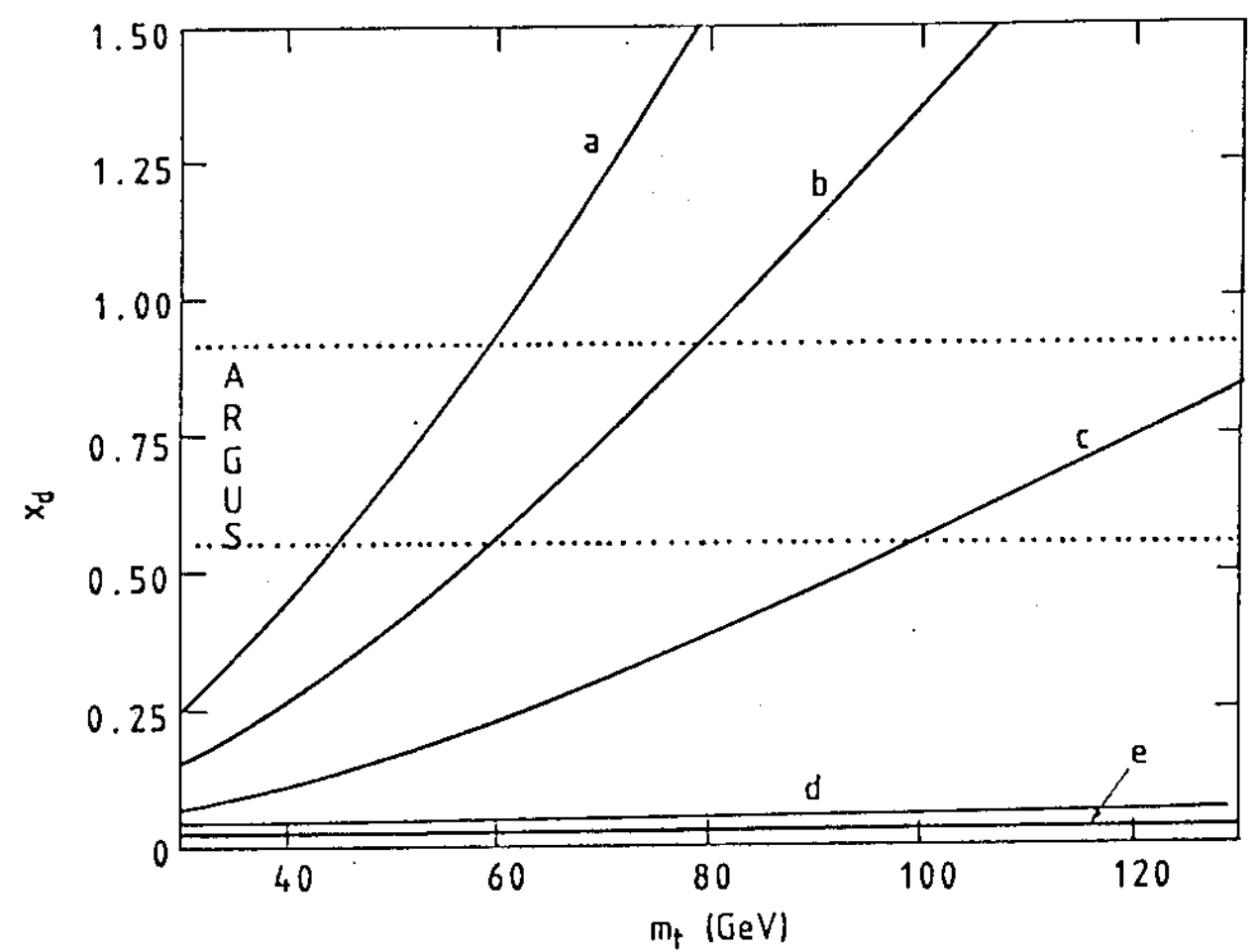

Fig. 9 Predicted values of $\mathrm{B}_{\mathrm{d}}^{0}-\overline{\mathrm{B}}_{\mathrm{d}}^{0}$ mixing in the standard model with three fermion families, as a function of the top quark mass, for different choices of the relevant parameters ${ }^{7}$. The ARGUS value [Eq. (1.1)] for $r_{d}$ is also shown, which translates to $x_{d}=$ $0.73 \pm 0.18$ by the relation $r_{d}=x_{d}^{2} /\left(2+x_{d}^{2}\right)$. All curves are for $\rho<0.9$ [or $\Gamma(b-u) / \Gamma(b-c)<0.08]$ where $\rho$ is defined by $\lambda \rho=$ $\left|v_{b u}\right| /\left|v_{b c}\right|$ with $\lambda \simeq 0.221$. In the curves $(a)-(e)$ we use the following values of the parameters $F=B_{B}^{\frac{1}{2}} f_{B}, \cos \phi$ and $T=$ $\tau_{B}\left|v_{b c}\right|^{2 / 10^{-15} s:}$

a. $F=180 \mathrm{MeV}, T=3.5$, and $\cos \phi=(\cos \phi)_{\mathrm{min}}$ (the largest negative value of $\cos \phi$ allowed by the $|\varepsilon|$ constraint). This curve gives the lower bound $m_{t}>45 \mathrm{GeV}$ quoted in the text.

b. $F=140 \mathrm{MeV}, T=3.5$, and $\cos \phi=(\cos )_{\min }$.

c. $F=140 \mathrm{MeV}, T=2.9$ and $\cos \phi=0$. This curve corresponds to about the central prediction of the standard model, and leads to the statement in the text that probably $m_{t}>90 \mathrm{GeV}$.

d. $F=100 \mathrm{MeV}, \mathrm{T}=2.3$ and $\cos \phi=(\cos \phi)$ (the 1 argest positive value of $\cos \phi$ allowed by the $|\varepsilon|$ constraint).

e. $F=100 \mathrm{MeV}, T=3.5$ and $\cos \phi=(\cos \phi)_{\max }$. 


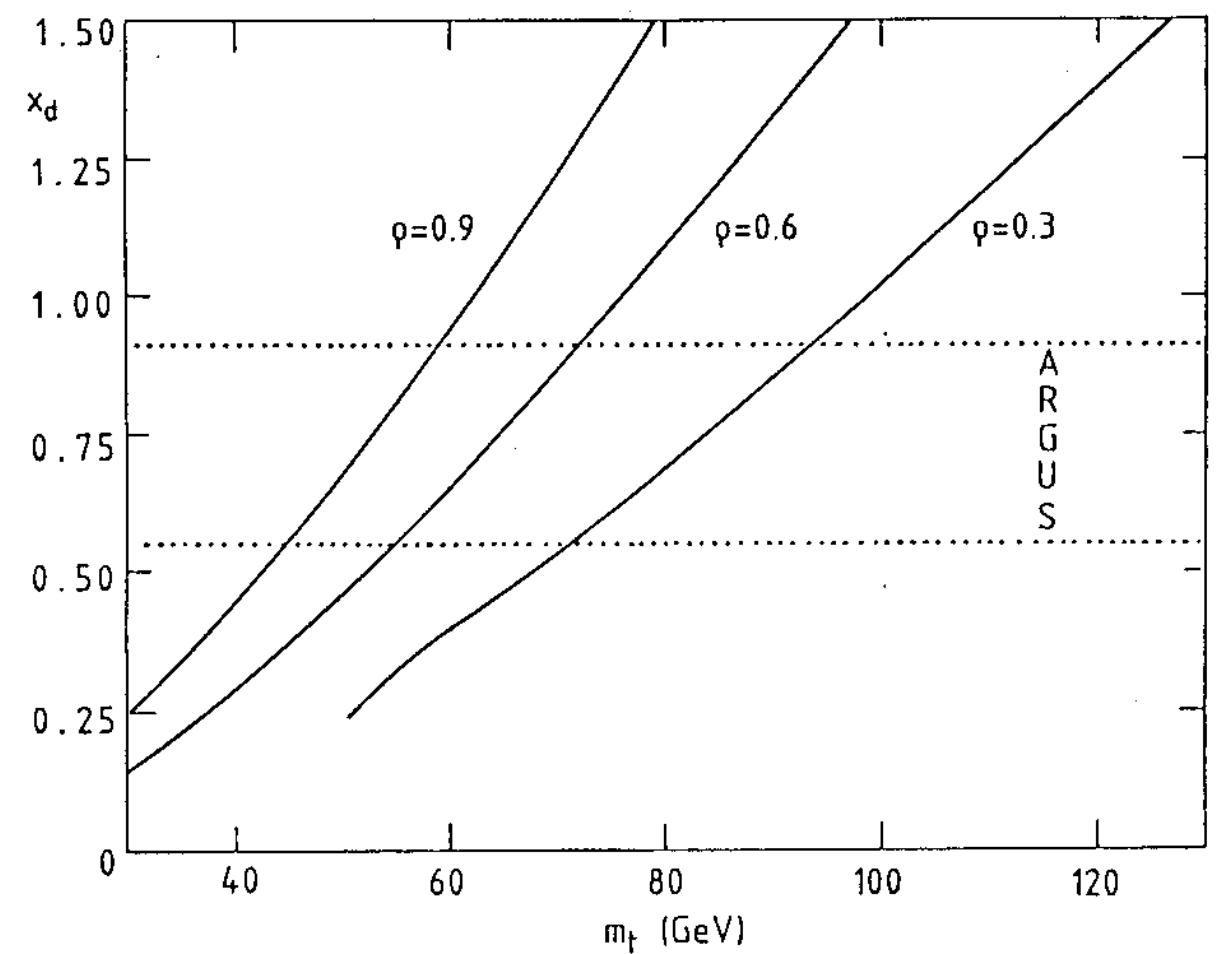

Fig. 10 Maximum value of $x_{d}$ compatible with the standard model with three fermion families as a function of the top quark mass, for different values of $\rho 7 . \rho$ is related to $R=\Gamma(b \rightarrow u) / \Gamma(b \rightarrow c)$ $(\rho=0.9,0.6$ and 0.3 correspond to $R \approx 0.08,0.04$ and 0.009 ). The $\rho=0.9$ curve corresponds to curve (a) of Fig. 9, i.e., ${ }_{B}^{\frac{1}{2}} f_{B}=180 \mathrm{MeV}, \tau_{B}\left|v_{b c}\right|^{2}=3.5 \times 10^{-15} \mathrm{~s}$, and $\cos \phi=(\cos \phi)_{\mathrm{min}}$ is the appropriate minimum value allowed by the $|\varepsilon|$ constraints, which changes with $\rho$. 


\section{8. $B^{0} \bar{B}^{0}$ MIXING BEYOND THE STANDARD MODEL}

In this section we consider the effect on $B^{0}-\bar{B}^{0}$ mixing (and in general on flavour mixing) of a number of simple generalizations of the standard model. We will discuss in detail the case of supersymmetry, charged Higgses, left-right models ${ }^{7}$. In all the generalizations of the standard model considered in the following the CKM suppression of $r_{d}$ versus $r_{s}$ is maintained. Thus the prediction of $r_{s}$ near unity is not altered in these minimal models of new physics. This is a reflection of the fact that the experimental absence of flavour changing neutral currents imposes really stringent constraints. Thus all reasonable models are constructed in such a way as to preserve the validity of the GIM mechanism and of the CKM hierarchy of couplings.

We first consider minimal models of supersymmetry broken softly by gravity 42 . The dominant additional contribution to flavour mixing from virtual exchange of supersymmetric particles arises from box diagrams with gluino and down squark exchange, as shown in Fig. 1la. In fact, it is well known ${ }^{42}$ that flavour changing couplings $\widetilde{g}-q-\widetilde{q}$ (where $\tilde{\mathrm{g}}$ and $\widetilde{q}$ denote gluinos and squarks) are induced by charged Higgsino exchange in (left-handed) down squark self mass diagrams (Fig. 1lb). The corresponding down squark mass squared matrix acquires a component proportional to $\mathrm{M}^{2}$ :

$$
m^{2} \tilde{d}_{L}=\mu^{2} \|+M_{d}^{2}-|c| V+M_{u}^{2} V
$$

where $M_{d}$ are the down, up quark mass matrices. We are systematically neglecting non-diagonal terms in the ${ }^{\prime}$ mass squared matrix connecting left and right squarks, since these presumably small effects can well be absorbed, at this exploratory state, in our ignorance of the other parameters. The parameter $|c|$ plays a crucial role in the following in that the interesting effect vanishes for $c=0$ and increases rapidly with $|c|$. $c$ is related to the Yukawa couplings of the Higgs particles and is expected to be of $O(1)$. The really relevant parameter is not $c$ but $\mathrm{cm}_{t}^{2} / \mathrm{m}_{\mathcal{J}}^{2}$. However, for $\mathrm{m}_{t} \sim \mathrm{mg}_{\mathrm{g}}$ in various simple models $\mathrm{s}^{42},|\mathrm{c}|$ is found to be in the range 0.1 to 1 . But there are no stringent arguments that prevent somewhat larger values of $|c|$. Thus, it appears reasonable to set $|\mathrm{c}|=1$ if we are interested in obtaining an estimate of how large the supersymetric contribution can be at most for a given value of $m_{t}$. Results for $|c|=\frac{1}{2}$ will also be given.

The gluino box diagrams lead to the following expressions ${ }^{42}$ for $\Delta M_{a b}$ (QCD correction factors are not included at this stage):

$$
\Delta M_{a b}^{\tilde{g}}=\frac{\alpha_{s}^{2}}{54 m_{\tilde{g}}^{2}} B_{B} f_{B}^{2} m_{B} \sum_{i j} \lambda_{a b}^{i} \lambda_{a b}^{j} S_{i j}\left(z_{i}, z_{j}\right)
$$

Here $\alpha_{s}$ is the QCD coupling at $a_{*}$ scale of order $\mathrm{m}_{\tilde{q}} \approx \mathrm{M}_{\mathrm{W}}$. We set in the following $\alpha_{s} \approx 0.12$. $\lambda_{\mathrm{ab}}=\mathrm{v}_{\mathrm{i} a}{ }^{*} \mathrm{v}_{\mathrm{ib}}$ and the index $\mathrm{i}$ can be $\mathrm{u}$, $\mathrm{c}$ or $t$. $s_{i j}\left(z_{i}, z_{j}\right)$ is a kernel given by

$$
S_{i j}=11 K_{i j}+4 I_{i j}
$$

with

$$
\begin{aligned}
& K_{i j}=\frac{1}{z_{i}-z_{j}}\left[\frac{z_{i}^{2} \ln z_{i}}{\left(1-z_{i}\right)^{2}}+\frac{1}{1-z_{i}}-(i \leftrightarrow j)\right] \\
& I_{i j}=\frac{1}{z_{i}-z_{j}}\left[\frac{z_{i} \ln z_{i}}{\left(1-z_{i}\right)^{2}}+\frac{1}{1-z_{i}}-(i \leftrightarrow j)\right]
\end{aligned}
$$




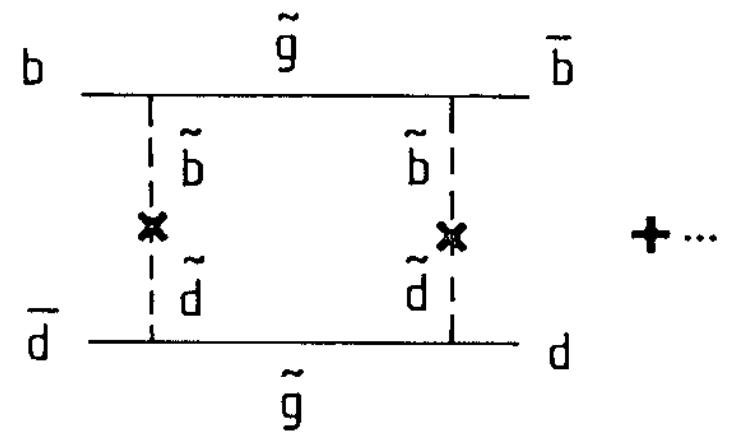

(a)

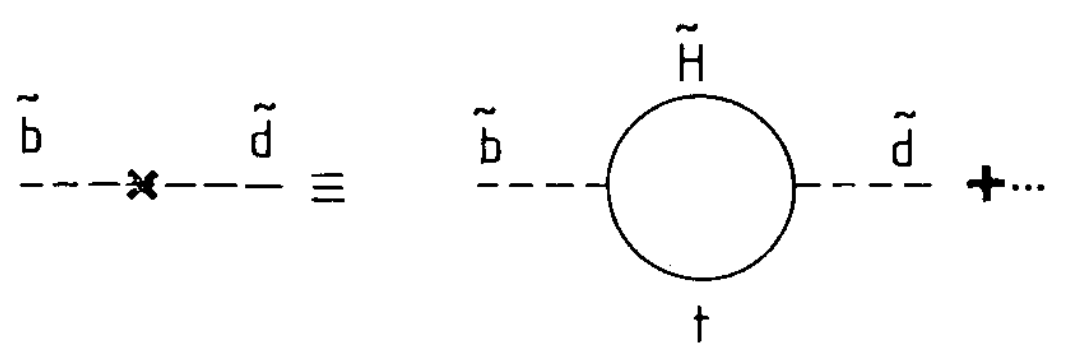

(b)

Fig. 11 a) Box diagram with gluino and squark exchange.

b) Charged Higgsino and top quark exchange contributing to flavour non-diagonal b to d (left-handed) squark mass matrix elements. 
where $z_{i}=m \tau_{i}^{2} / m_{\tilde{g}}^{2}\left(\widetilde{d}_{i}=\widetilde{d}, \tilde{s}, \tilde{b}\right.$ are the down type squarks $)$. In the excellent approximation $(0.1 \%)$ of neglecting all quark masses except $\mathrm{m}_{t}$, one has $\mathrm{m}^{2}=\mathrm{m}_{\mathrm{s}}^{2}=\mathrm{m}^{2}+|c| \mathrm{m}^{2}$; Then, by using the unitarity of the CKM matrix, one can reduce Eq. (8.2) to the simpler, more explicit form:

$x_{d}^{\tilde{g}}=\frac{\alpha_{s}^{2}}{54 m_{\tilde{g}}^{2}} \tau_{B_{d}} B_{B_{d}} f_{B_{d}}^{2}\left|V_{t b} V_{t d}^{*}\right|^{2} \Delta S_{t}\left(m_{\tilde{j}}, m_{\tilde{b}}, m_{\tilde{g}}\right)$

where

$$
\Delta S_{t}=S_{33}\left(z_{3}, z_{3}\right)+S_{11}\left(z_{1}, z_{1}\right)-2 S_{13}\left(z_{1}, z_{3}\right)
$$

other supersymmetric contributions arising from wino or Higgsino exchange are much suppressed, since $\alpha_{s}$ is relatively large and up squarks are nearly degenerate in mass.

In order to obtain numerical results, we need to input values for the gluino and squark masses. Taking into account the UAl 1 imits as presented in Ref. 43 , we consider $\pi_{\tilde{g}} \geqslant 55-60 \mathrm{GeV}$ and $\pi r y 260-70 \mathrm{GeV}$. In Figs. 12 we plot ${ }^{7}$, for different values of the relevant parameters, the ratio $x^{\text {TOT }} / x^{S T}$ of the total resulting $x^{\text {TOT }}=x^{S^{T}}+x^{\tilde{g}}$ for the $B_{d}^{0}$ sesons

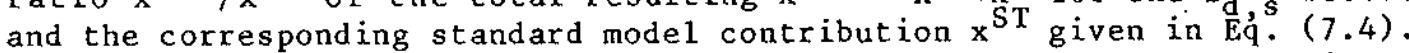
This ratio is particularly interesting because it is independent of the values of $\mathrm{CKM}$ angles, of $\mathrm{B}_{\mathrm{B}} \mathrm{f}_{\mathrm{B}}^{2}$ and of $\tau_{B}$. We see that the contribution of gluino box diagrams can indeed be important as $r_{d}$ grows quadratically with $x_{d}$ at small $x_{d}$. It is to be stressed, however, that the supersymmetric effect drops very rapidly with $\mathrm{m}_{\mathrm{g}}$ and my. The dependence on $|\mathrm{c}|$ is also very sharp (the ratio $x \widetilde{g} / x^{S T}$ is approximately of the form $R=|c|$ $\left.\mathrm{f}\left(|\mathrm{c}| \mathrm{m}_{t}^{2}\right)\right)$. Note that the requixement of positivity of $\mathrm{m}_{\mathrm{b}^{2}}=\mathrm{m}_{\mathrm{g}}{ }^{2}-|\mathrm{c}| \mathrm{m}_{\mathrm{t}}^{2}$ implies an upper bound on $m_{t}$ for given values of $\mathrm{mr}^{2}$ and $|c|$. The drop at large $m_{t}$ of the supersymetric contribution visible in Figs. 12 is due to this upper bound.

The effects of supersymmetry on $\mathrm{B}^{0}-\overline{\mathrm{B}}^{0}$ mixing were also studied in Ref. 44. Unfortunately, their numerical results are marred by a sign error. Their Eq. (5) should be changed of sign on the right-hand side. As a consequence, in their Eq. (10) the coefficient $-16 / 27$ becomes $-8 / 27$ (and $+16 / 9$ becomes $-4 / 9$ ) so that the result given here and in Refs. 42 is reproduced. Once this error is corrected, we agree with them when the small values of $c$ they use are taken into account.

In conclusion, supersymmetry could in principle explain the large value of $r_{d}$ at moderate values of $m_{t}$, but only if gluino and squark masses are close to the present experimental bounds. On the other hand, we have checked that in all cases studied here the supersymmetric contribution to $\mathrm{M}_{\mathrm{K}_{\mathrm{I}}}{ }^{-\mathrm{M}_{\mathrm{S}}}$ is completely negligible. Also, in the presence of supersymmetric effects, the constraints arising from the observed value of $|\varepsilon|$ are further relaxed.

It is interesting to recall that the same flavour changing mechanism that contributes to $\mathrm{B}^{0}-\overrightarrow{\mathrm{B}}^{0}$ mixing can lead to sizeable branching $\mathrm{ratios}$ for decay rates of the types $b \rightarrow s \gamma$ or $b \rightarrow s g$ which could be large enough to be observed, as discussed in ReFs. 45. 


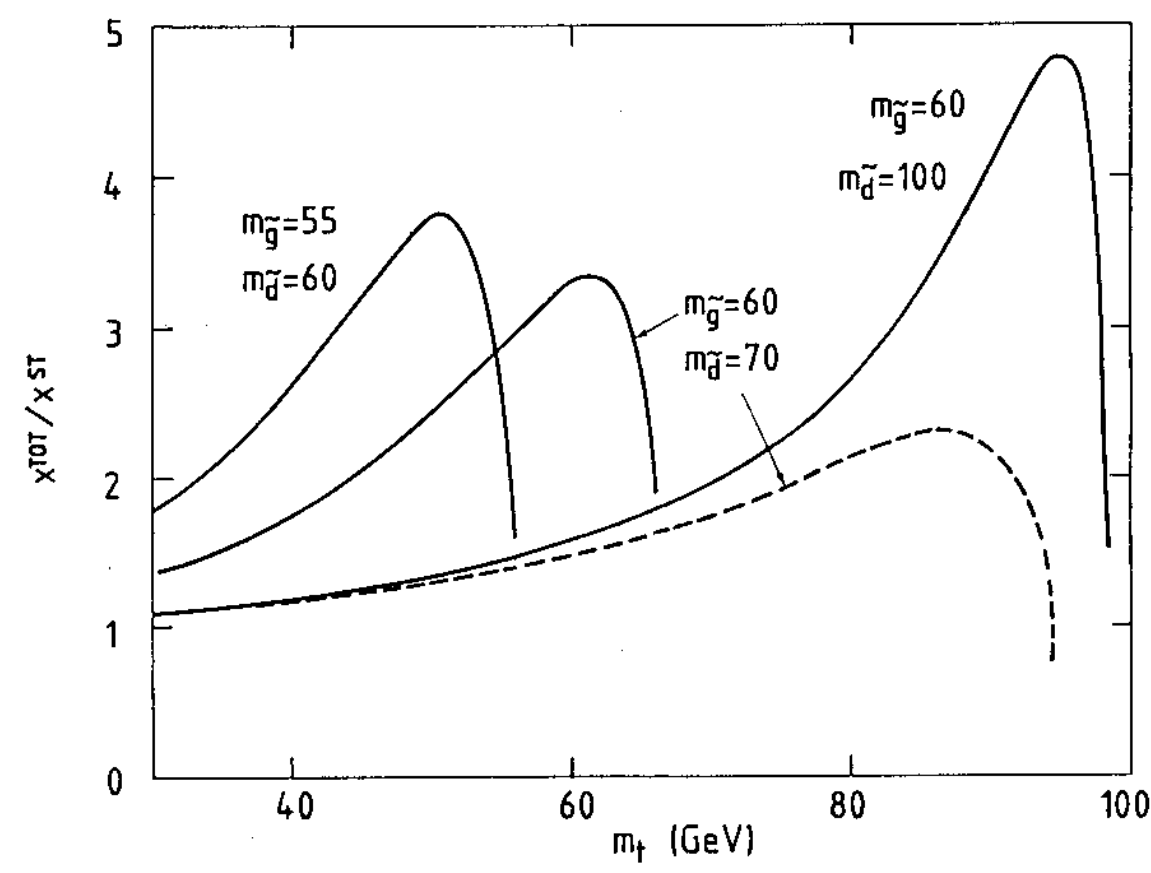

Fig. 12 The ratio $x^{T O T} / x^{S T}$ for $B_{d}^{0}$ or $B_{s}^{0}$ mesons, where $x^{T O T}=x^{S T} \widetilde{g}^{\widetilde{g}}$ is the sum of the standard term, Eq. (7.1), plus the supersymmetric contribution, given in Eq. ( 8.5$)$. The solid lines correspond to $|c|=1$, while the dashed 1 ine is computed for $|c|=\frac{1}{2}$, where $\mid \begin{aligned} & c \\ & c\end{aligned}$ is defined in Eq. (8.1). The gluino and down squark masses (in GeV) are indicated on the curves 7 . 
We now consider the contribution of charged Higgses. In all the models based on supersymmetry, physical charged Higgs bosons are also predicted. Additional box diagrams contributions to $B^{0}-\bar{B}^{0}$ mixing obtained by replacing one or both of the $W$ lines by virtual charged Higgses are therefore also present. We shall now discuss these terms briefly. Of course, charged Higgs contributions to $\mathrm{B}^{0}-\overline{\mathrm{B}}^{0}$ mixing can occur independently of supersymetry, so that the following discussion is of more general validity. In Refs. 46,47 the box diagrams with charged Higgs exchange were computed, and their implications for $B^{0}-B^{0}$ mixing were discussed. In a model with two Higgs doublets, the relevant couplings of charged Higgses to quarks are given by

$$
H=\frac{g_{2} H^{t}}{2 \sqrt{2} M w} u_{R} \frac{v_{d}}{v_{u}} M_{V} V d L+\cdots
$$

where $u$ and $d$ indicate up- and down-type quarks respectively, $g_{2}$ is the $\mathrm{SU}(2)$ weak gauge coupling, $\mathrm{M}$ is the diagonal up quark mass matrix, and $\mathrm{V}$ is the CKM matrix. $v_{d}$ and $v_{u}$ are the Higgs vacuum expectation values of the Higgs doublets giving mass to the down-type and up-type quarks respectively; they are proportional to the down and up quark masses via the corresponding Yukawa coupling constants. The ellipsis indicates terms proportional to the down quark mass matrix which can be neglected in comparison with the top quark contribution. The exact result for $x_{d}$ can be obtained from Ref. 46. An approximate form (adequate for the scope of the present discussion) of the correction to the standard result, due to charged Higgs exchange, is given by Ref. 47

$$
x_{d} \simeq x_{d}^{s T}\left[1+\frac{1}{4}\left(\frac{v_{d}}{v_{u}}\right)^{4}\left(\frac{m_{t}}{m_{H}}\right)^{2}\right]
$$

The limits of validity of this simple approximation obtained for $m_{t}<m_{H}$, and neglecting the box diagram with one Higgs and one $W$ propagator, are discussed in Ref. 46. For $\mathrm{m}_{\mathrm{t}} \lesssim \mathrm{m}_{\mathrm{H}}$, the exact expression gives $\mathrm{x}_{\mathrm{d}}$ larger, by about $10 \%$ or less. One sees, for example, that for $m_{H} \sim M_{W}$ and $m_{t}$ as low as $\mathrm{m}_{\mathrm{t}} \sim 30$ to $40 \mathrm{GeV}$ a ratio $\mathrm{v}_{\mathrm{d}} / \mathrm{v}_{\mathrm{u}} \sim 2$ to 4 is sufficient to yield a possible explanation for the observed value of $r_{d}$. Such values for the ratio of vacuum expectation values are perfectly admissible (see Ref. 46 for current limits). However, we note that the perhaps appealing requirement of using the duplication of doublets to decrease the differences among the Yukawa couplings of Higgs bosons to quarks would lead to $v_{d} / v_{u}<1$ since up quarks are on the average heavier than down quarks. Also in minimal models of supersymetry broken by gravity $v_{u}>v_{d}$ as a consequence of the running of the Yukawa up-couplings on the way from $M_{p l}$ down to $M_{W}$ induced by the relatively heavy top quark.

We now review $B^{0}-\bar{B}^{0}$ mixing in the context of minimal left-right symmetric models 48 as discussed in Refs. 49 and 50 and add some observations. By minimal models we mean both a single multiplet of Higgs bosons coupled to quarks (transforming as $\left(\frac{1}{2}, \frac{1}{2}, 0\right)$ of $S U(2){ }_{L} \otimes S_{(2)}(2) U(1)$ and spontaneous CP violation with identical or complex conjugate. CKM matrices for left-handed and right-handed quarks. It is well known that the contribution to the $K_{L}-K_{S}$ mass difference arising from (right-handed) $W_{R}$ exchange in the box diagrams are much larger than for the ordinary (lefthanded) $W_{L}$ of the same mass 50 . Requiring that the short-distance contribution to $\Delta \mathrm{m}_{\mathrm{K}}$ does not exceed the experimental value leads in fact to a strong limit on the $W_{R}$ mass: $M_{W_{R}}>1.5$ to $2.5 \mathrm{TeV}$. There are also additional contributions to $\Delta \mathrm{m}_{\mathrm{K}}$ arising from tree-level diagrams with flavour 
changing Higgs exchange, with masses $\mathrm{M}_{\mathrm{H}}$ order $\mathrm{M}_{\mathrm{W}_{\mathrm{R}}}$. Thus a lower 1 imit on $\mathrm{M}_{\mathrm{H}}$ of several TeV can be derived.

In the approximation of neglecting the small mixing $\xi_{\text {LR }}$ between left and right components in the charged weak boson mass matrix, the charged current term in the Hamiltonian can be written

$$
H=\frac{g_{2}}{\sqrt{2}}\left\{W_{L}^{+\mu} \bar{u} V_{L} \gamma_{\mu} \frac{1-\gamma_{S}}{2} d+W_{R}^{+\mu} \bar{u} V_{R} \gamma_{\mu} \frac{1+\gamma_{r}}{2} d\right\}+h^{\prime c} c_{(8.9)}
$$

where $v_{L}$ and $v_{R}$ are the CKM matrices for left- and right-handed quarks respectively, which we assume to be the same in the following. The total resulting box diagram contributions to $x_{d, s}$, including the standard contribution $x^{L L}$ from $W_{L}$ exchange plus the $W_{R}$ and unphysical scalar exchange terms, is given by:

$$
\begin{aligned}
x_{d, s}^{\text {TOT }} & =x_{d, s}^{L L}\left\{1+\frac{3}{2} \frac{M_{w_{L}}^{2}}{M_{W_{R}}^{2}}\left(\frac{m_{B S, s}^{2}}{\left(m_{b}+m_{d, s}\right)^{2}}+\frac{1}{6}\right) \frac{1}{\eta_{Q C D}} \frac{\eta}{A(\eta)} .\right. \\
& {\left.\left[\eta_{t 1}^{L R}\left(\frac{4-\eta}{1-\eta}+\frac{4-2 \eta+\eta^{2}}{(1-\eta)^{2}} \ln \eta\right)+\eta_{t 2}^{L R} \ln \frac{M_{W_{L}}^{2}}{M_{W_{R}}^{2}}\right]\right\} }
\end{aligned}
$$

Here $\eta_{\mathrm{OCD}}$, $\eta_{\text {and }} \mathrm{A}(\eta) / \eta$ are defined in Eqs. $(6.12),(5.21)$ and $(5.22)$ and $\eta_{t 1} L_{R} \eta_{t 2}{ }^{2} 1.8$ are additional QCD correction factors. From the above formula, one can immediately derive the important results that

a) the amount of $B^{0}-\bar{B}^{0}$ mixing is not very much altered with respect to the standard model result, once the constraints on $\mathrm{M}_{\mathrm{W}_{\mathrm{R}}}$ imposed by the observed value of $\Delta \mathrm{m}_{\mathrm{K}}$ are taken into account.

b) The sign of the effect for $m_{t} \lesssim 250 \mathrm{GeV}$ goes in the direction of reducing the amount of mixing with respect to the standard model if one assumes that, after diagonalization of the mass matrix, all right-handed quark mass eigenvalues are positive in the CKM phase choice (as discussed in $\operatorname{Ref} .49$ this is not really necessary).

c) The absolute value of the correction slowly decreases with $m_{t}$.

In fact, compared with the $\mathrm{K}^{0}-\overline{\mathrm{K}}^{0}$ system, the importance of the diagrams with left-right exchange is much decreased because $\left(m_{B}^{2} /\left(m_{b}+m_{d, s}\right)^{2}\right) \sim 1$ is small in comparison with $\left(m_{K}^{2} /\left(m_{s}+m_{d}\right)^{2}\right)$. Moreover, the dominant contribution for the $k^{0}-\bar{K}^{0}$ system arises from charm exchange. Then $\ell n \rightarrow \rightarrow l n \mathrm{~m}_{\mathrm{c}}^{2} / \mathrm{m}_{\mathrm{W}}^{2}$ in Eq. (8.10) and the corresponding term is quite large. As a consequence, for values of $\mathrm{M}_{\mathrm{W}_{\mathrm{R}}}$ large enough to be compatible with $\Delta \mathrm{m}_{K}$, the corresponding correction for $B^{0}-\bar{B}^{0}$ mixing is small.

Similarly, the Higgs exchange term is also negative, and smaller than for the $\mathrm{K}^{0}-\overline{\mathrm{K}}^{0}$ system. Neglecting QCD corrections, one obtains: 


$$
x_{d, s}^{H i g g s}=-x_{d, s}^{L L} \frac{\sqrt{2} 6 \pi^{2}}{G_{f} m_{H}^{2}}\left(\frac{m_{B d, s}^{2}}{\left(m_{b}+m_{d, s}\right)^{2}}+\frac{1}{6}\right) \frac{\eta}{A|\eta|}
$$

Since the additional logarithmic enhancement due to the relative smal1ness of the charm mass is not present in this case, the absolute value of the Higgs correction $c$ an be larger than that arising from the box diagrams.

\section{CONCLUSION}

The ARGUS result on $B_{d}^{0}-\bar{B}_{d}^{0}$ mixing can be accommodated in the standard model with three families provided that $m_{t}$ is large. The first important experimental check is to measure the mixing parameter $r_{s}$ for the $B_{s}^{0}$ mesons. If $r_{s}$ is not nearly maximal, the most obvious possibility is a fourth family. On the other hand, simplest extensions of the standard model tend to preserve the Kobayashi-Maskawa pattern. Thus if $r_{s}$ is near one, then lower values of $\mathrm{m}_{t}$ can be allowed as an effect of charged Higgs exchange or of supersymmetry. In the former case, one needs relatively light charged-Higgs bosons and a ratio of vacuum expectation values $v_{d} / v_{u}>1$ (which is perhaps counter-intuitive and not what minimal supersymmetry would predict), where $v_{d} u$ are the vacuum expectation values of the Higgs doublet giving masses to the $d$ or $u$ type of quarks respectively. In the latter case, the supersymmetric contribution can only be important if the parameters are somewhat stretched to the most favourable situation. In particular squarks and gluinos should have relatively small masses, close to the experimental lower bounds. Finally, minimal left-right-symmetric models do not lead to important effects for $B^{0}-\bar{B}^{0}$ mixing if the bounds from kaon mixing are taken into account. 


\section{REFERENCES}

1. H. Albrecht et al. (ARGUS Collaboration), DESY Report DESY-87-029 (1987).

2. C. Albajar et a1. (UAl Collaboration), Phys. Lett. 186B (1987) 247.

3. I. Mannelli, Proceedings of the 1987 Int. Symposium on Lepton and Photon Interactions at High Energies, Hamburg (1987).

4. S.-L. Wu, these proceedings.

5. J.S. Hagelin, Nucl. Phys. B193 (1981) 123;

I.I. Bigi and A.I. Sanda, Phys. Rev. D29 (1984) 1393;

V. Barger and R.J.N. Phillips, Phys. Lett. 1438 (1984) 259;

L. -L. Chau and W.Y. Keung, Phys. Rev. D29 (1984) 592;

E.A. Paschos and U. Türke, Nucl. Phys. B243 (1984) 29;

A. Al i, DESY Reports 85-107 (1985) and 86-108 (1986);

A.J. Buras, W. Slominski and H. Steger, Nuc1. Phys. B245 (1984) 369;

For a review, see also:

L. -L. Chau, Physics Reports 95 (1983) 1.

6. J. Ellis, J.S. Hagelin and S. Rudaz, CERN preprint TH.4679 (1987);

L.-L. Chau and W.Y. Keung, Univ. of California at Davis Report UCD-87-02 (1987);

A. Datta, E.A. Paschos and U. Türke, Univ. of Dortmund Report DO-TH-87/9 (1987);

D.F. Donoghue et al., SIN-PR-87-05 (1987);

H. Harari and Y. Nir, Stanford Report SLAC-PUB-4341 (1987);

W.S. Hou and A. Soni, Univ, of Pittsburgh Report PITT-87-06 (1987);

L. Angelini et al, Univ. of Bari Report BARI-GT/2-87 (1987);

V.A. Khoze and N.G. Ural'tsev, Leningrad Report 1290 (1987).

7. G. Altarelli and P. Franzini, CERN preprint TH.4745 (1987).

8. J. Maalampi and M. Roos, Univ, of Helsinki Report HU-TFT-87-12 (1987).

9. V. Barger, T. Han, D.V. Nanopoulos and R.J.N. Phillips, Univ. of Wiscons in Report MAD/TH/87-12 (1987);

J.R. Cudell et al., Univ. of Wisconsin Report MAD/PH/352 (1987).

10. A. Ali, Proceedings of the UCLA Workshop for a $B \bar{B}$ factory, DESY Report (1987).

11. See, for example:

R.L. Kingsley, Phys. Lett. 63B (1976) 329;

I. Bigi and A.I. Sanda, Phys. Lett. B171 (1986) 320 .

12. S.L. Glashow, J. Iliopoulos and L. Maiani, Phys. Rev. D2 (1970) 1285 .

13. M.K. Gaillard and B.W. Lee, Phys. Rev. Dlo (1975) 897.

14. For a recent review of CP violation, see:

J.F. Donoghue, B.R. Holstein and G. Valencia, Int. Journal of Mod. Phys. 2 (1987) 319.

15. L. Maiani, Proceedings of the Int. Symposium on Lepton and Photon Interactions at High Energies, Hamburg (1977). 
16. T. Kobayashi and M. Maskawa, Prog. Theor. Phys. 49 (1973) 652.

17. L. Wolfenstein, Phys. Rev. Lett. 51 (1983) 1945.

18. Particle Data Group, Phys. Lett. 170B (1986) 1.

19. G. Altarel1i, N. Cabibbo, G. Corbo, L. Maiani and G. Martinelli, Nuc1. Phys. B208 (1982) 365.

20. D.M. Ritson, Proceedings of the 23rd Int. Conference on High Energy Physics, Berkekey (1986).

21. E.W. Thorndike and R.A. Poling, University of Rochester preprint, UR-1022 (1987), submitted to Physics Reports.

22. W. Schmidt-Parzefall, Proceedings of the Int. Symposium on Lepton and Photon Intereactions at High Energies, Hamburg (1987).

23. S. Behrends et al., (CLEO Collaboration), Phys. Rev. Lett. 59 (1987) 407 .

24. B. Grinstein, M.B. Wise and N. Isgur, Phys. Rev. Lett. 56 (1986) 298.

25. See, for example;

K. Schubert, Proceedings of the EPS Conference on High Energy Physics, Uppsala, Sweden (1987).

26. T. Inami and C.S. Lim, Prog. Theor. Physics 65 (1981) 297.

27. M.K. Gaillard and B.W. Lee, Phys. Rev. Lett. 33 (1974) 108;

G. Altarelli and L. Maiani, Phys. Lett. B52 (1974) 351.

28. T. Schaad et al. (MARK II Collaboration), Phys. Lett. 160B (1985) 188.

29. M.B. Gavela et al., presented by 0 . Pène at the Proceedings of the EPS Int. Conference on High Energy Physics, Uppsala, Sweden (1987).

30. C. Bernard et a1., private communication by A. Soni.

31. J.F. Donoghue, E. Golowich and B.R. Holstein, Phys. Lett. 119B (1982) 412 .

32. A. Pich and E. de Rafae1, Phys. Lewtt. 158B (1985) 477.

33. W.A. Bardeen, A.J. Buras and J.-M. Gerard, MPI Report MPI-PAE(PTH 22/87 (1987).

34. E.V. Shuryak, Nuc1. Phys. B198 (1982) 83.

35. V.L. Chernyak et al., Sov. J. Nuc1. Phys. 38 (1983) 773.

36. T.A. Aliev et a1., Sov. J. Nu8cl. Phys. 38 (1983) 936.

37. S. Narison, CERN preprint TH.4746 (1987).

38. C.A. Dominguez and N. Paver, DESY Report 87-060 (1987).

39. J. Adler et a1. (MARK III Collaboration), SLAC-PUB-4343 (1987). 
40. L.J. Reinders, H. Rubinstein and S. Yazaki, Physics Reports 127 (1985) 1 .

41. K. Kleinknecht and B. Renk, Z. Phys. C34 (1987) 209.

42. M.J. Duncan and J. Trampetic, Phys. Lett. 134B (1984) 439;

J.-M. Gexard et al., Phys. Lett. 140B (1984) 349;

J.-M. Gerrard et al., Phys. Lett. 141B (1984) 79; Nucl. Phys. B253 (1985) 93;

P. Langacker and B. Sathiapalan, Phys. Lett. 144B (1984) 395; Phys; Lett. "144B (1984) 401;

M. Dugan, B. Grinstein and L. Hal1, Nuc1. Phys. B255 (1985) 413;

A. Bouquet, J. Kaplan and C.A. Savoy, Phys. Lett. 148B (1984) 69.

43. S. Geer, Proceedings of the EPS Int. Conference on High Energy Physics, Uppsala, Sweden (1987).

44. S. Bertolini, F. Borzumati and A. Masiero, Carnegie Mellon Report CMU-HEP 87-07 (1987).

45. S. Bertolini, F. Borzumati and A. Masiero, Carnegie-Mellon Univ. Reports CMU-HEP 86-19 (1986) and 87-03 (1987);

N.G. Deshpande et a1., Univ. of Oregon Reports OITS-346 (1986).

46. L.F. Abbott, P. Sikivie and M.B. Wise, Phys. Rev. D21 (1980) 1393;

See also the recent paper by:

S.L. Glashow and E.J. Jenkins, HUTP-87/A047 (1987).

47. G.G. Athanasiu, P.J. Franzini and F.J. Gilman, Phys. Rev. D32 (1985) 3010 .

48. J.C. Pati and A. Salam, Phys. Rev. D10 (1974) 275;

R.N. Mohapatra and J.C. Pati, Phys. Rev. Dll (1975) 566, 2558;

R.N. Mohapatra and G. Senjanovic, Phys. Rev. D12 (1975) 1502, Phys. Rev. Lett. 44 (1980) 912; Phys. Rev. D23 (1981) 165.

49. G. Ecker and W. Grimus, Nuc1. Phys. B258 (1985) 3281 and Z. Phys. C30 (1986) 293.

See, also:

R. Decker and U. Turke, Z. Phys. C26 (1984) 117.

50. G. Beal1, M. Bander and A. Soni, Phys. Rev. Lett. 48 (1982) 848;

G. Ecker, W. Grimus and H. Neufeld, Phys. Lett. 127B (1983) 365;

R.N. Mohapatra, G. Senjanovic and M.D. Tran, Phys. Rev. D28 (1983)

546 ;

F.J. Gilman and M.H. Reno, Phys. Rev. D29 (1984) 937. 